Original Paper http://ajol.info/index.php/ijbcs http://indexmedicus.afro.who.int

\title{
Phytoécologie des faciès à Marantaceae de la forêt de Lossi (Congo, Brazzaville)
}

\author{
Victor KIMPOUNI
}

École Normale Supérieure, Université Marien Ngouabi; B.P. 237, Brazzaville, République du Congo.

E-mail :vkimpouni@yahoo.com

\section{RÉSUMÉ}

La forêt à Marantaceae de Lossi, au Sud de la Haute Sangha, est étudiée suivant un transect de $5000 \mathrm{~m}$ traversant trois faciès (terre ferme, périodiquement inondé et marécageux). L'inventaire couvre 2,5 ha pour les arbres de $10 \leq \mathrm{dhp}<70 \mathrm{~cm}$ et 25 ha pour ceux de $\mathrm{dhp} \geq 70 \mathrm{~cm}$. 178 espèces sont recensées pour une quarantaine de familles et 1168 arbres. On relève 903 arbres de $10 \leq \mathrm{dhp}<70 \mathrm{~cm}$ et $265 \mathrm{de} \mathrm{dhp} \geq 70 \mathrm{~cm} ; 164$ espèces de $10 \leq \mathrm{dhp}<70 \mathrm{~cm}$ contre 56 pour le $\mathrm{dhp} \geq 70 \mathrm{~cm}$ dont 14 y sont spécifiques. La composition floristique révèle peu d'espèces communes. Les indices de diversité biologique révèlent un haut degré de diversification, une faible richesse floristique et une prépondérance des arbres de petit diamètre. L'indice des valeurs d'importance couplé au coefficient d'abondance-dominance montre, suivant le type forestier, une dominance de quelques familles. Les valeurs des données phytoécologiques sont inférieures à celles connues des forêts denses d'Afrique centrale. Nonobstant un important cortège régénératif, l'apport spécifique dénote une insuffisance de régénération naturelle, vu la dominance des taxons de sous-bois. Leur affinité aux forêts atlantiques de l'aire nigéro-camerouno-gabonaise, dont Scyphocephalium mannii et Dacryodes sp. sont les marqueurs, est décelée. Les sarcochores dominent et l'endozoochorie est le principal mode de dissémination. Le type foliaire mésophile, synonyme d'hélophytes, prédomine.

(C) 2017 International Formulae Group. All rights reserved.

Mots clés : Congo, indice de diversité biologique, forêt à Marantaceae, diversité floristique, phytoécologie, élément phytogéographique.

\section{Phytoecology of Marantaceae facies in the Lossi forest (Congo, Brazzaville)}

\begin{abstract}
Phytoecologic of Marantaceae facies in the Lossi forest (Congo, Brazzaville). The Lossi Marantaceae forest, in the South of Haute Sangha, is studied along a transect of $5000 \mathrm{~m}$ crossing three facies (land, periodically flooded and swampy). The inventory covers 2.5 ha for trees of $10 \leq \mathrm{dhp}<70 \mathrm{~cm}$ and 25 ha for those of dhp $\geq 70 \mathrm{~cm} .178$ species are recorded for about forty families and 1168 trees. There are 903 trees of $10 \leq \mathrm{dhp}<70 \mathrm{~cm}$ and $265 \mathrm{dbh} \geq 70 \mathrm{~cm} ; 164$ species of $10 \leq \mathrm{dhp}<70 \mathrm{~cm}$ against 56 for the dhp $\geq 70 \mathrm{~cm}$ of which 14 are specific. The floristic composition reveals few common species. Biodiversity indices show a high degree of diversification, low floristic richness and a preponderance of small diameter trees. The index of importance values coupled with the coefficient of abundance-dominance shows, depending on the forest type, a dominance of a few families. The values of the phytoecological data are lower than those known from the dense forests of Central Africa. Notwithstanding a large regenerative procession, the specific contribution
\end{abstract}


indicates the lack of natural regeneration, given the dominance of undergrowth taxa. Their affinity to the Atlantic forests of the Niger-Cameroonian-Gabonese area, including Scyphocephalium mannii and Dacryodes sp. which are the markers, is detected. Sarcochores dominates and endozoochories is the main mode of dissemination. The leaf type mesophylle, synonymous of heliophytes, predominates.

(C) 2017 International Formulae Group. All rights reserved.

Keywords: Congo, biological diversity index, Marantaceae forest, floristic diversity, phytoecology, phytogéographic element.

\section{INTRODUCTION}

Le Congo s'étire de part et d'autre de l'équateur, entre les latitudes $4^{\circ} \mathrm{N}$ et $5^{\circ} \mathrm{S}$ et les longitudes 11 et $19^{\circ} \mathrm{E}$; couvre 342000 $\mathrm{km}^{2}$ (Vennetier, 1977). Deux principaux écosystèmes se mettent en évidence, la forêt et la savane. La couverture forestière correspond à $60-65 \%$ de son aire, alors que la savane, deuxième composante, occupe le reste du territoire, soit 35 à 40\% (De Wasseige et al., 2012, 2014).

La forêt congolaise représente $10 \%$ des forêts du continent, soit $12 \%$ de forêts denses d'Afrique centrale (De Wasseige et al., 2012, 2014). Cette couverture forestière étant "discontinue », les trois massifs sont d'inégale superficie. Au Sud, les massifs du Mayombe (1,2 million ha) et du Chaillu (3,3 millions ha); le massif du Nord Congo (15,3 millions ha) dans la partie septentrionale (De Wasseige et al., 2012, 2014 ; Kimpouni et al., 2013, 2014). Ce dernier couvre deux faciès écologiques basés sur la nature du substrat. La forêt inondée de la Basse Sangha $(20 \%)$ et celle de terre ferme de la Haute Sangha (31\%). Au plan phytogéographique, la flore $\mathrm{du}$ Nord-Congo démarque deux districts phytogéographiques et subit les influences des éléments Atlantique de l'aire nigérocamerouno-gabonaise au Sud, camerounocongolais au Nord et congolais à l'Est (Kouka, 2006 ; Kimpouni et al., 2013). Suivant la chorologique africaine, notamment du centre d'endémisme guinéo-congolais, la flore du Congo dans son ensemble se développe dans le couloir du « Sangha River» défini par White (1986) et reste très mal connue.

Les acquis des études sur la flore et la végétation du Congo, dénotent que les massifs du Mayombe et du Chaillu, dont l'exploitation industrielle remonte aux années 1928 pour le premier, sont les mieux explorés par rapport à celui du Nord Congo dont la valorisation est très récente. $\mathrm{Au}$ niveau actuel des connaissances sur la diversité végétale, le massif du Nord Congo est le plus riche et le plus diversifié des trois (De Wasseige et al., 2012, 2014 ; Kimpouni et al., 2013, 2014). Au regard des potentialités économiques prouvées par les travaux d'inventaire botanique de Rollet (1964), de Bégué (1967), du CTFT (1974), de Hecketsweiler (1990), de Hecketsweiler et al. (1991), la végétation et la flore du Nord-Congo font l'objet de plusieurs investigations relatives à la mise en valeur des ressources ligneuses et non ligneuses. En fonction des exigences et des buts poursuivis, ces études restent fragmentaires et très générales pour plusieurs d'entre elles. Dans ce contexte, elles intéressent généralement des aires assez larges et ne rapportent presque rien sur les spécificités relatives à certains types d'habitats, où s'exercent des activités particulières. C'est le cas du site de Lossi qui abrite le sanctuaire de gorille de plaine. Les études catégorielles sur les forêts à Marantaceae étant rares, les données existantes sont relatives au parc national d'Odzala-Kokoua et portent sur les groupements phytosociologiques et la catégorisation de ces types forestiers (Kouka, 2001, 2006 ; Senterre, 2005). Les données phytoécologiques sur les forêts à Marantaceae qui constituent le faciès le plus représentatif de la végétation de la Haute Sangha (Hecketsweiler, 1990) sont encore insignifiantes et très parcellaires. Nonobstant les travaux menés au Parc national d'Odzala, aucune donnée n'est disponible sur la structure, la dynamique et le fonctionnement de cet écosystème. 
Les caractéristiques écologiques de la diversité floristique attestent une stabilité fonctionnelle du système, au sein duquel la complexité d'interactions dynamiques est telle qu'une variation ou perturbation du milieu n'a qu'un faible impact (Bond et Chase, 2002 ; Giller et O’Donovan, 2002 ; Wallington et al., 2005 ; Caldeira et al., 2005; Mikkelson, 2009). Ce stade de développement intègre une étroite corrélation entre richesse spécifique et performance ou stabilité fonctionnelle de l'écosystème, traduisant généralement son état de développement. Le niveau de connaissances écologiques acquis sur les rapports entre le degré de diversité floristique et la stabilité fonctionnelle des écosystèmes, montre que leur évolution est concomitante. Ainsi, le faible degré de diversité (homogénéité floristique) d'un système n'implique pas toujours son instabilité, et inversement, l'état de stabilité ne correspond pas à une forte hétérogénéité (diversité floristique élevée) de l'écosystème (Loreau et al., 2002 ; Bond et Chase, 2002 ; Giller et O'Donovan, 2002 ; Wallington et al., 2005 ; Caldeira et al., 2005 ; Mikkelson, 2009).

Afin d'apporter un accent à l'amélioration des connaissances sur les forêts à Marantaceae, cette étude cible la collecte des données phytoécologiques de la forêt de la Haute Sangha et particulièrement les faciès de Lossi. Le choix porté sur la forêt à Marantaceae de Lossi est justifié par le fait qu'elle abrite d'importantes populations de grands primates, base de la création du sanctuaire de gorille de Lossi en 1999.

Ce travail, qui est une contribution à l'amélioration des connaissances sur la flore forestière du Congo, se focalise sur la caractérisation des faciès forestiers à Marantaceae du Sud de la Haute Sangha. Il est centré sur une analyse de la phytodiversité ligneuse et l'influence des éléments phytogéographiques. Les hypothèses de travail se déclinent ainsi qu'il suit: les forêts du Sud de la Haute Sangha se développent sous la dépendance de l'élément Atlantique de l'aire nigéro-camerouno-gabonaise, d'une part, et, dominées floristiquement par les
Burseraceae et les Euphorbiaceae, d'autre part; le niveau de régénération naturelle dans les faciès forestiers à Marantaceae est faible ; la forêt à Marantaceae présente concomitamment un haut degré de diversité spécifique ligneuse et un faible nombre d'individus.

\section{MATÉRIEL ET MÉTHODES \\ Présentation su site d'étude}

La forêt de Lossi $\left(00^{\circ} 14^{\prime} 09^{\prime \prime} \mathrm{N}, 14^{\circ}\right.$ 30' 12" E), située au Sud-Ouest du parc national Odzala-Kokoua, est un maillon du massif forestier de la Haute Sangha dont la végétation se compose principalement de forêts denses humides semi décidues, au sein desquelles se caractérise le faciès de forêts claires. Les forêts claires, définies par SaintAubin (1963) comme des formations végétales à essences émergentes très isolées « clair-plantées », sans sous-bois ni individus de diamètre intermédiaire, dominent une haute strate herbacée (2 à $3 \mathrm{~m}$ ) essentiellement de Marantaceae et de Zingiberaceae (Kouka, 2006; Van Loon, 2009; Gillet, 2013 ; Kimpouni et al., 2013). Ces faciès sont les plus représentatifs de cet écosystème et les moins étudiés de la flore congolaise.

D'une superficie de 35000 ha, le sanctuaire de gorille de plaine de Lossi est créé en 1999 (Figure 1). De ce fait, la forêt de Lossi bénéficie d'un statut de protection à travers la loi forestière. La végétation du Congo étant planitiaire, le gradient d'altitude de la forêt de Lossi oscille entre 200 et $400 \mathrm{~m}$ (Kimpouni et al., 2013). Cet écosystème évolue sous un climat équatorial de type AW3 suivant la classification de Köppen (1936). Ses principales caractéristiques sont une pluviométrie moyenne annuelle de $1800 \mathrm{~mm}$, des températures oscillant autour de $25{ }^{\circ} \mathrm{C}$ toute l'année. Les précipitations s'étalent sur toute l'année avec un net ralentissement aux mois de juillet et août. Les pics des précipitations sont observés entre les mois de mars et mai pour le premier, et le second entre octobre et novembre (Samba, 2008 ; Samba et Nganga, 2011). 


\section{Méthode d'inventaire}

La flore du sanctuaire de Lossi est étudiée suivant un transect botanique de $5 \mathrm{~km}$ de long. Cet inventaire est mené dans des placettes de 0,1 ha et couvre une aire de $5 \mathrm{~m} \mathrm{x}$ $5000 \mathrm{~m}$ (soit 2,5 ha) pour les ligneux des classes de diamètre $10 \leq \mathrm{dhp}<70 \mathrm{~cm}$. Pour ceux dont le dhp $\geq 70 \mathrm{~cm}$, la superficie couverte est de 25 ha (soit $50 \mathrm{~m} \times 5000 \mathrm{~m}$ ) correspondant à 25 relevés de 1 ha. Le choix des deux classes de diamètre de l'inventaire est associé au diamètre moyen d'exploitabilité (DME), oscillant autour de $70 \mathrm{~cm}$, pour la majorité des essences. L'orientation du transect étant corrélée à la distribution et l'occupation du site par les différentes « familles » de gorilles y évoluant, il traverse successivement trois faciès forestiers tenant à la nature du substratum :

- La forêt de terre ferme ( 1 ha pour les arbres de $10 \leq \mathrm{dhp}<70 \mathrm{~cm}$ et 10 ha pour ceux de $\mathrm{dhp} \geq 70 \mathrm{~cm}$;

- La forêt périodiquement inondée ( 1 ha pour les arbres de $10 \leq \mathrm{dhp}<70 \mathrm{~cm}$ et 10 ha pour ceux de dhp $\geq 70 \mathrm{~cm})$;

- et la forêt marécageuse $(1 / 2$ ha pour les arbres de $10 \leq \mathrm{dhp}<70 \mathrm{~cm}$ et 5 ha pour ceux de dhp $\geq 70 \mathrm{~cm}$ ).

Dans ces bandes, tous les arbres de dhp (diamètre à hauteur de poitrine) supérieur ou égal à $10 \mathrm{~cm}$ à $1,30 \mathrm{~m}$ au-dessus du sol sont identifiés (Dallmeier, 1992; Martin, 2004). Pour chaque sujet, on note la position par rapport à la médiane et à l'origine du transect. La largeur de la bande d'inventaire étant de 5 et $50 \mathrm{~m}$, elle est traversée longitudinalement et à équidistance des bornes par le layon de comptage (soit à 2,5 et $25 \mathrm{~m}$ de part et d'autre). Suivant les classes de diamètre, les arbres sont regroupés en 6 classes pour les arbres de $10 \leq \mathrm{dhp}<70 \mathrm{~cm} ; 5$ classes de diamètre dont une classe regroupant les ligneux faiblement représentés des classes supérieures ou égales à 11 , pour les arbres de dhp $>70 \mathrm{~cm}$. Au cours de cet inventaire floristique, un millier d'échantillons d'herbiers est récolté et déposé à l'herbarium national du Congo (IEC). Ce matériel est traité suivant la classification taxinomique de
l'APG III (Angiosperm phylogeny group, 2009).

Les paramètres écologiques suivis dans la forêt de Lossi sont la densité (D), les types foliaires, les types de diaspores et la dissémination, le type phytogéographique, l'abondance-dominance, la surface terrière (ST), le rapport ST/D, d'une part, et, d'autre part, la densité relative (DR), la fréquence relative (FR) et l'indice de diversité relative ; les indices de diversité biologique: le coefficient de similarité de Sørensen qui avantage deux fois plus l'importance à la présence et l'indice de Simpson qui privilégie l'importance des espèces rares.

L'indice de valeur d'importance des espèces (IVI) et des familles (FIV) renseigne, pour chaque taxon, sur le nombre d'individus, la distribution et le niveau d'occupation spatiale des troncs: surface terrière (Nusbaumer et al., 2005 ; Vroh et al., 2010).

Le coefficient d'abondance-dominance (densité) des taxa inventoriés est défini suivant une échelle de valeurs liée au nombre d'individus par hectare (Lacoste et Salanon, 2006), d'une part, et tenant compte du modèle de distribution, d'autre part. Dans le cas de Lossi, le calcul des coefficients se base sur des sections de transect appartenant au même type forestier. Ainsi, l'échelle de valeur se présente comme suit: $(+)$ moins d'un d'arbre.ha ${ }^{-1},(1)$ de 1 à 3 arbres.ha ${ }^{-1},(2)$ de 4 à 6 arbres.ha ${ }^{-1}$, (3) de 7 à 9 arbres.ha $^{-1}$, (4) de 10 à 12 arbres.ha ${ }^{-1}$ et (5) plus de 12 arbres.ha ${ }^{-1}$.

La contribution de types de dimensions foliaires est basée sur la classification de Raunkiaer (1934). Les types retenus sont: leptophylles (Lepto) $<0,2 \mathrm{~cm}^{2}$, nanophylles (Nano) de 0,2 à $2 \mathrm{~cm}^{2}$, microphylles (micro) de 2 à $20 \mathrm{~cm}^{2}$, mésophylles (Méso) de 20 à $200 \mathrm{~cm}^{2}$, macrophylles (Macro) de 2 à $20 \mathrm{dm}^{2}$, mégaphylles (Méga) $>20 \mathrm{dm}^{2}$.

Les types de dissémination des diaspores sont établis suivant Dansereau et Lems (1957) et Évrard (1968). Les types reconnus sont les ptérochores (Ptéro), diaspores munies d'appendices aliformes; les pogonochores (Pogono), diaspores pourvues d'appendices plumeux ou soyeux ; sclérochores (Scléro), diaspores non charnues 
relativement légères ; les sarcochores (Sarco), diaspores totalement ou partiellement charnues; les barochores (Baro): diaspores non charnues et lourdes; les ballochores (Ballo), diaspores expulsées par la plante ellemême.

Les types de distribution phytogéographique sont fondés sur la chorologique de la flore africaine (White 1979, 1986). Selon cette classification, les éléments suivant sont ainsi reconnus: (1) élément à large distribution [pantropicale (Pant), afro-américaine (Afam), afromalgache (Afma), afrotropicale (Aftr)], (2) élément de liaison [Guinéo-Zambézienne (GZ)], (3) élément endémique [GuinéoCongolais (GC); Centro-guinéenne $(\mathrm{Cg})$ y compris le subcentre d'endémisme BasGuinéen (Bg)].

\section{Analyse des données}

Les paramètres phytoécologiques suivis pour analyser les données sont :

- la fréquence $=$ nombre de relevés où le taxon est présent / nombre total des relevés ;

- la densité = nombre de taxons (ou arbres) / unité de surface ;

- la surface terrière $\left(\mathrm{ST}\right.$ en $\left.\mathrm{m}^{2} \cdot \mathrm{ha}^{-1}\right)=\pi \cdot \mathrm{R}^{2} ; \mathrm{R}$ $=$ rayon du tronc à dhp, constante $\pi=3,14$;
- la fréquence relative $(\mathrm{FR}$ en $\%)=100 \mathrm{x}$ (densité du taxon / $\sum$ fréquences des taxons) ;

- la densité relative $(\mathrm{DR}$ en $\%)=100 \mathrm{x}$ (densité du taxon $/ \sum$ densités totales des taxons) ;

- la dominance relative (DoR en $\%)=100 x$ (ST du taxon / $\sum$ ST totales des taxons) ;

- l'indice de diversité relative (IDR en \%) = 100 x (nombres d'espèces de la famille / nombre total d'espèces) ;

- l'indice de valeur d'importance (IVI) $=\mathrm{FR}+$ DR + DoR; Quant à la FIV (indice de valeur d'importance des familles), elle est égale à la somme des IVI des espèces ;

- le coefficient de similarité de Sørensen (\%) $=100 \times(2 \mathrm{C} /(\mathrm{A}+\mathrm{B}))$ avec $\mathrm{A}=$ nombre de spécimens du $1^{\text {er }}$ relevé; $\mathrm{B}=$ nombre de spécimens du $2^{\mathrm{e}}$ relevé; $\mathrm{C}=$ nombre de spécimens communs aux deux relevés;

- l'indice de diversité biologique de Simpson

$$
\mathrm{D}=1-\sum_{\mathrm{i}=1}^{\mathrm{S}} \mathrm{ni}(\mathrm{ni}-1) / \mathrm{n}(\mathrm{n}-1)
$$

avec $\mathrm{s}=$ nombre total d'espèces $; \mathrm{pi}=$ abondance relative de l'espèce $\mathrm{i}(\mathrm{ni} / \mathrm{n})$; ni $=$ nombre d'individus de l'espèce $\mathrm{i}$; $\mathrm{n}=$ nombre total des individus toutes espèces confondues.

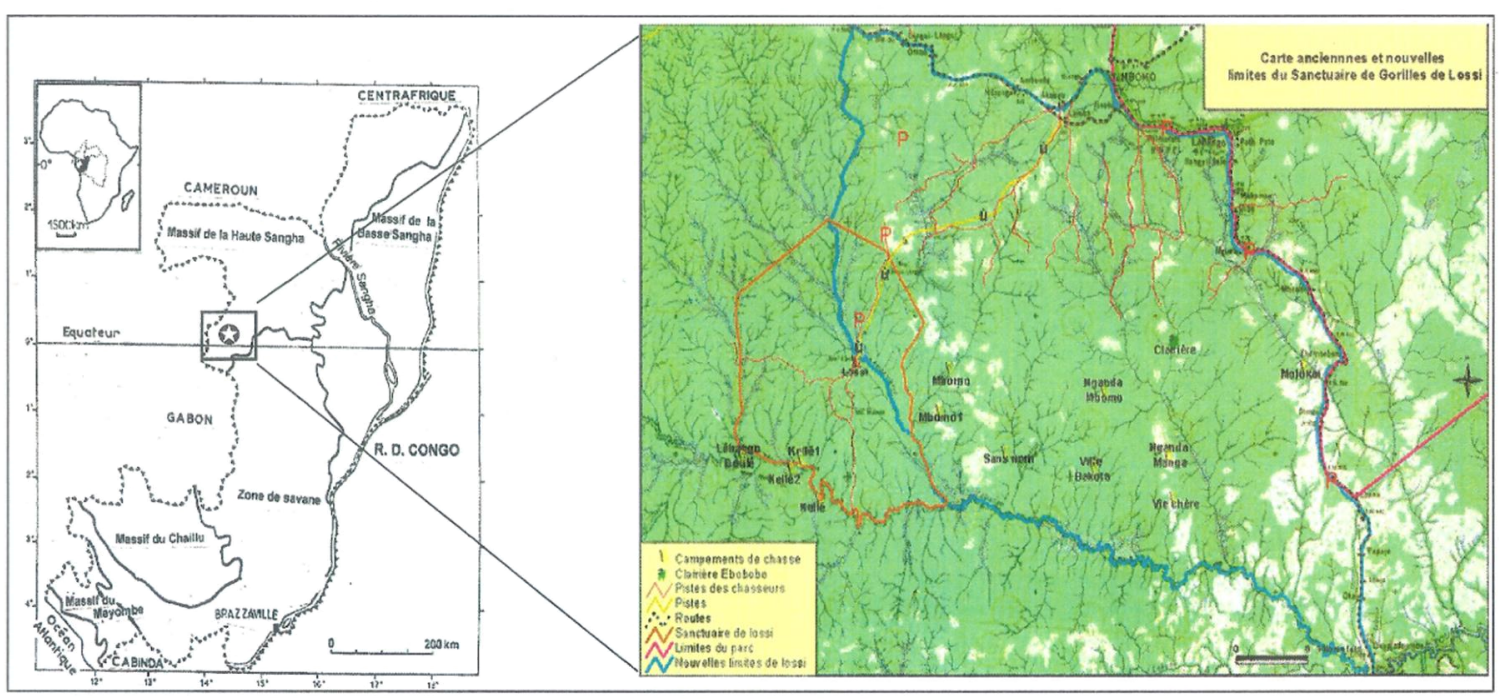

Figure 1 : Localisation du sanctuaire de gorilles de Lossi dans le massif forestier de la HauteSangha. 


\section{RÉSULTATS}

\section{Analyse taxonomique de la flore}

L'inventaire floristique global recense 1168 arbres correspondant à 903 ligneux de $10 \leq \mathrm{dhp}<70 \mathrm{~cm}$ et $265 \mathrm{de} \mathrm{dhp} \geq 70 \mathrm{~cm}$ (Tableau 1). Une quarantaine de familles associant les 178 espèces sont identifiées. Suivant les critères de l'inventaire, 164 espèces sont répertoriées dans les deux classes de diamètre dont seulement 42 espèces (21\%) dans la classe de dhp $\geq 70 \mathrm{~cm}$. Les 14 autres espèces sont recensées exclusivement dans la classe de dhp $\geq 70 \mathrm{~cm}$ (Tableau 1).

\section{Diversité floristique de la forêt de Lossi}

Dans les trois faciès forestiers, les densités sont particulièrement élevées pour les arbres de $10 \leq \mathrm{dhp}<70 \mathrm{~cm}$. Le faciès marécageux est le plus densifié avec 204 arbres recensés sur $1 / 2$ ha. La densité est respectivement de 334 et 365 arbres.ha $^{-1}$ dans le faciès de terre ferme et périodiquement inondé. Cependant, elle est faible dans les relevés des ligneux de $\mathrm{dhp} \geq 70 \mathrm{~cm}$ (Tableau 1). La densité est de 9 arbres.ha $^{-1}$ dans les facies périodiquement inondé et marécageux, contre 13 individus.ha ${ }^{-1}$ dans celui de terre ferme.

Pour les ligneux de10 $\leq \mathrm{dhp}<70 \mathrm{~cm}$

Les densités de la diversité floristique de la forêt de Lossi sont présentées dans la Figure 2.

- Dans la forêt de terre ferme (Figure 2A), la densité par espèce est comprise entre 1 et 51 arbres.ha $^{-1}$, soit une moyenne de 3,18 \pm 0,52 arbres.ha ${ }^{-1}$. 74\% des espèces recensées ont 1 à 3 arbres.ha ${ }^{-1}$. En fonction du nombre d'espèces (spectre brut), les Fabaceae devancent nettement les Euphorbiaceae, les Rubiaceae, les Malvaceae, les Annonaceae et les Burseraceae. Cependant, le spectre pondéré (Figure 2B) met distinctement en valeur les Burseraceae face aux Fabaceae. Le faciès de terre ferme serait caractérisé par les Burseraceae (77 arbres.ha ${ }^{-1}$ ), notamment Dacryodes buettneri ( 9 arbres.ha $^{-1}$ ) et Santiria trimera (51 arbres.ha' $\left.{ }^{-1}\right)$.
- Dans la forêt périodiquement inondé (Figure 2A), la densité varie de 1 à 31 ligneux.ha ${ }^{-1}$ (soit une moyenne de 3,4 $\pm 0,25$ arbres.ha $^{-1}$ par espèce). Des taxons inventoriés, plus du $1 / 3$ à 1 arbre.ha ${ }^{-1}$. le spectre brut met en évidence les Euphorbiaceae, Fabaceae et Rubiaceae. Par contre, l'analyse basée sur le nombre de pieds recensés met en relief, par ordre d'importance, les Burseraceae, les Fabaceae et les Euphorbiaceae (Figure 2B). Au regard des contributions associées aux familles dominantes, cette flore définirait une forêt mélangée (mixte) avec une prédominance des Euphorbiaceae par rapport aux Burseraceae et aux Fabaceae. Les principales essences sont Plagiostyles africana (31 arbres.ha $\left.{ }^{-1}\right)$ et Gymnanthes inopinata (13 arbres.ha ${ }^{-1}$ ) pour les Euphorbiaceae; Dacryodes buettneri (14 arbres.ha ${ }^{-1}$ ) et Santiria trimera (19 arbres.ha ${ }^{-1}$ ) pour les Burseraceae; et enfin Berlinia grandiflora ( 8 arbres.ha ${ }^{-1}$ ) pour les Fabaceae.

- Dans le type marécageux (Figure 2A) la densité oscille de 1 à 23 arbres au $1 / 2$ ha, avec 3,58 $\pm 0,44$ arbres au $1 / 2$ ha en moyenne. Dans ce faciès un peu plus de la moitié des espèces recensées a une densité de 1 arbre au $1 / 2$ ha. Le nombre d'espèces révèle dans l'ordre les Fabaceae, les Rubiaceae et les Malvaceae. Par ailleurs, le spectre pondéré ressort les Sapindaceae devant les Rubiaceae et enfin les Fabaceae (Figure 2B). Cette forêt mélangée est caractérisée par Allophyllus africanus (23 arbres par $1 / 2$ ha), Eriocoelum microspermum (20 arbres par $1 / 2$ ha), Diospyros canaliculata (18 arbres par $1 / 2$ ha), Berlinia grandiflora (13 arbres par $1 / 2$ ha) et Rothmannia lujae (10 arbres par $1 / 2$ ha). Rappelons que Allophyllus africanus et Eriocoelum microspermum sans être des essences typiques de cet écosystème, sont les témoins de leur perturbation.

\section{Pour les ligneux de dhp $\geq 70 \mathrm{~cm}$}

- Dans la forêt de terre ferme la densité varie de 0,1 à 6 arbres.ha ${ }^{-1}$ avec une moyenne par espèce de $0,44 \pm 0,06.95 \%$ de l'ensemble des taxons ont moins d'un arbre.ha ${ }^{-1}$. Le spectre brut ressort une nette dominance des Fabaceae devant les Myristicaceae et les 
Irvingiaceae (Figure 2C). Cependant, le spectre pondéré est en faveur des Fabaceae suivies des Burseraceae (Figure 2D). Les taxons à forte contribution sont Pentaclethra eetveldeana $\left(0,7\right.$ pieds.ha $\left.{ }^{-1}\right)$, Pentaclethra macrophylla (1 pieds.ha- $\left.{ }^{-1}\right)$ pour les Fabaceae ; Dacryodes buettneri (1,2 pieds.ha $\left.{ }^{-1}\right)$ pour les Burseraceae et Petersianthus macrocarpus $\left(0,9\right.$ pieds.ha $\left.{ }^{-1}\right)$ pour Les Lecythidaceae.

- Dans la forêt périodiquement inondée, les espèces recensées ont entre 0,1 et 1 arbre.ha $^{-1}$ soit une moyenne de 0,2 $\pm 0,04$ arbre.ha $^{-1}$ (Figure 2D). Cet inventaire ressort que $95 \%$ des espèces ont moins d'un arbre.ha' ${ }^{1}$. La diversité spécifique dégage les Fabaceae, les Myristicaceae, les Burseraceae et les Sapindaceae (Figure 2C). Quant au nombre d'individus inventoriés, les Burseraceae se démarquent devant les Myristicaceae, les Fabaceae, les Lecythidaceae, les Irvingiaceae et les Rubiaceae. les espèces caractéristiques sont Dacryodes buettneri (1 pied.ha $\left.{ }^{-1}\right)$ et Canarium schweinfurthii (0,5 pied.ha $\left.{ }^{-1}\right)$; Scyphocephalium mannii (0,5 pied.ha $\left.{ }^{-1}\right)$; Petersianthus macrocarpus (0,5 pieds.ha $\left.{ }^{-1}\right)$.

- Dans le type marécageux (Figure 2D), les taxons recensés ont une densité comprise entre 0,2 et 4,2 arbres.ha ${ }^{-1}$, soit une moyenne de $0,7 \pm 0,16$ arbre.ha ${ }^{-1}$. Dans ce faciès, $92 \%$ d'espèces ont moins d'un arbre.ha ${ }^{-1}$. Suivant la diversité spécifique, les Rubiaceae dominent les Myristicaceae et les Fabaceae (Figure 2C). En fonction du nombre d'individus inventoriés, les Rubiaceae devancent les Myristicaceae et les Anacardiaceae. Les taxons caractéristiques sont Mitragyna stipulosa (4,2 pieds.ha $\left.{ }^{-1}\right)$, Coelocaryon botryoides $\left(0,8\right.$ pieds.ha $\left.{ }^{-1}\right)$, Scyphocephalium mannii $\left(0,8\right.$ pieds.ha $\left.{ }^{-1}\right)$ et Pseudospondias microcarpa $\left(0,8\right.$ pieds.ha $\left.{ }^{-1}\right)$.

\section{Analyse de l'abondance-dominance}

L'abondance-dominance de la diversité floristique de la forêt de Lossi est présentée dans la Figure 3.

\section{Ligneux de $10 \leq \mathrm{dhp}<70 \mathrm{~cm}$}

○ Le coefficient d'abondance-dominance révèle que près de $3 / 4$ des espèces (soit
$73,33 \%$ ) ont entre 1 et 3 arbres.ha ${ }^{-1}$, dans la forêt de terre ferme (Figure 3A). Les taxons les mieux représentés, pris ensemble, ont une contribution de 7,07\%. Ce groupe est constitué de Dacryodes buettneri et Strombosia grandiflora (coefficient d'abondance-dominance égale à 3), Caloncoba welwitschii, Lannea welwitschii (coefficient d'abondance-dominance égale à 4), Santiria trimera et Dialium pachyphyllum (coefficient d'abondance-dominance égale à 5). Ce résultat montre une insuffisance quantitative des espèces donnant les arbres de première grandeur de la forêt dense d'Afrique centrale. La conséquence inéluctable se traduirait par une mauvaise régénération naturelle dudit faciès forestier ;

- Dans la forêt périodiquement inondée (Figure 3A), plus de $2 / 3$ des taxons (soit $69,81 \%$ ) sont faiblement représentés. Les meilleurs taux d'abondance-dominance sont associés à Polyalthia suaveolens, Rinorea oblongifolia, Dichostemma glaucescens et Cola acuminata (avec un coefficient d'abondance-dominance de 3), Santiria trimera, Plagiostyles africana, Allophyllus africanus, Gymnanthes inopinata, Dacryodes buettneri et Berlinia grandiflora (avec un coefficient d'abondance-dominance de 5). Comme dans la forêt de terre ferme, on note une prédominance des taxons généralement confinés au sous-bois et ceux traduisant les perturbations du milieu;

- Dans la forêt marécageuse (Figure 3A), les taxons dont le coefficient d'abondancedominance varie entre 1 et 3 arbres par $1 / 2$.ha représentent les $2 / 3$ de l'inventaire, soit $77 \%$. Les taxons s'exprimant le mieux sont Mitragyna stipulosa, Cola acuminata, Pseudospondias microcarpa (coefficient d'abondance-dominance de 3), Rothmannia lujae (coefficient d'abondance-dominance de 4), Allophyllus africanus, Eriocoelum microspermum, Berlinia grandiflora et Diospyros canaliculata (coefficient d'abondance-dominance de 5). L'ensemble de ces taxons assure une contribution de $15 \%$. Outre Mitragyna stipulosa qui est une espèce 
caractéristique des milieux marécageux dans la région et de première grandeur, tout le reste, formant la grande majorité, donne généralement des petits arbres n'atteignant pas les $40 \mathrm{~cm}$ de dhp. Ce sont des espèces dominées de la strate arbustive et/ou du sousbois.

\section{Ligneux de dhp $\geq 70 \mathrm{~cm}$}

- Dans le faciès forestier de terre ferme, la majorité des taxons a un coefficient d'abondance-dominance de moins d'un arbre.ha $^{-1}$ (Figure 3B). La contribution de ces taxons est de $78,42 \%$. Les espèces qui ont un coefficient d'abondance-dominance élevé contribuent à hauteur de 21,58\%. Ce groupe est centré autour de Dacryodes buettneri, Pentaclethra macrophylla et Petersianthus macrocarpus avec un coefficient d'abondance-dominance de 1. Le comportement desdits taxons dans le faciès n'est autre que le reflet de l'inventaire des arbres de $10 \leq \mathrm{dhp}<70 \mathrm{~cm}$ dans la forêt de terre ferme ;

- Dans la forêt périodiquement inondée, notons que seules Dacryodes buettneri et Petersianthus macrocarpus ont un coefficient d'abondance-dominance de 1 (Figure 3B). Ces deux espèces représentent 21,98\% de l'inventaire. La majorité des taxons (78\%) a un coefficient d'abondance-dominance de moins d'un arbres.ha ${ }^{-1}$;

- Dans le faciès marécageux, seule Mitragyna stipulosa a un coefficient d'abondance-dominance de 2. Ce taxon inféodé au milieu marécageux a une contribution de $46,67 \%$, alors que la grande majorité $(77 \%)$ a moins d'un arbre.ha ${ }^{-1}$ (Figure 3B). Corrélation de similarité et indice de
diversité biologique
Le coefficient de similarité de Sørensen entre types forestiers révèle, pour les arbres de10 $\leq \mathrm{dhp}<70 \mathrm{~cm}$, que les faciès périodiquement inondé et de terre ferme sont à peine similaires $(56,2 \%)$. Par ailleurs, la similarité entre les facies périodiquement inondé et marécageux n'est à peine que de
$39,3 \%$. Il en est de même pour les faciès de terre ferme et marécageux avec $37,7 \%$ de similarité. Cette observation est aussi valable pour les arbres de $\mathrm{dhp} \geq 70 \mathrm{~cm}$. On note une similarité de $63,2 \%$ entre les faciès de terre ferme et le périodiquement inondé ; 37,5\% entre les faciès de terre ferme et marécageux, et enfin, $38,5 \%$ entre les types périodiquement inondé et marécageux. Cette donnée peut être un élément d'appréciation et de caractérisation $\mathrm{du}$ faciès, notamment le changement de la composition floristique d'un milieu à l'autre.

L'indice de diversité biologique de Simpson, pour les arbres de $10 \leq \mathrm{dhp}<70 \mathrm{~cm}$, montre que les différents types forestiers sont très faiblement diversifiés. Dans la forêt de terre ferme, il est de 0,22 et la contribution la plus forte est assurée par Santiria trimera; de 0,02 dans le type périodiquement inondé et de 0,05 dans le marécageux avec Allophyllus africanus comme principale essence. Pour les ligneux de $\mathrm{dhp} \geq 70 \mathrm{~cm}$, cet indice est de 0,2 dans le faciès marécageux et Mitragyna stipulosa est la principale essence; de 0,03 dans les types périodiquement inondé et de terre ferme. Les principales espèces sont Dacryodes buettneri et Petersianthus macrocarpus pour le premier et Dacryodes buettneri pour le second.

\section{Indice de valeur d'importance}

L'analyse des paramètres associés à la fréquence, la densité et la dominance donne en fonction du type forestier, les résultats suivants :

\section{Pour les ligneux de $10 \leq d h p<70 \mathrm{~cm}$}

- Dans la forêt de terre ferme, la fréquence relative oscille entre 0,5 et $4,3 \%$ avec $74 \%$ des taxons dont elle est inférieure à $1 \%$. La fréquence relative moyenne est de $1 \pm$ $0,16 \%$ Les principales espèces sont Santiria trimera et Strombosia grandiflora; la densité relative varie de 0,30 à $15,3 \%$ avec $74 \%$ d'espèces se situant en dessous de $1 \%$. Sa moyenne est de $1 \pm 0,07$ et Santiria trimera est l'espèce phare; la dominance relative des espèces est comprise entre 0,04 et $11,51 \%$ soit une moyenne de $1,05 \pm 0,16 \%$. $66 \%$ des 
espèces recensées ont densité relative inférieure à $1 \%$; l'importance relative est comprise entre 0,26 et $6,63 \%$. $65 \%$ des taxons présentent une valeur inférieure à $0,01 \%$. La principale essence est Santiria trimera; l'indice de valeur d'importance varie de 0,8 à $31 \%$ (soit une moyenne de $2,9 \pm 0,18 \%$ ) avec $31 \%$ des taxons présentant une valeur inférieure à $2 \%$; l'indice de valeur des familles varie de 0,8 à $56,2 \%$ avec une moyenne de 9,6 $\pm 1,09 \%$. Les taxons les plus en vue sont les Burseraceae $(56,2 \%)$, Fabaceae (38,1\%), Euphorbiaceae (18,9\%), Olacaceae (17,7\%), Annonaceae (17,3\%), Irvingiaceae $(15,9 \%)$, Anacardiaceae $(16,3 \%)$ et Achariaceae $(13,7 \%)$;

- Dans la forêt périodiquement inondée, la fréquence relative oscille entre 0,4 et $4 \%$ avec $69 \%$ des taxons dont elle est inférieure à $1 \%$. Sa moyenne est $0,9 \pm 0,05 \%$. Les principales espèces sont Santiria trimera, Plagiostyles africana et Dacryodes buettneri ; la densité relative varie de 0,3 à $9,2 \%$ (soit $1 \pm$ $0,08 \%$ en moyenne). $70 \%$ d'espèces ont une fréquence relative en dessous de $1 \%$. Les espèces mises en évidence sont Santiria trimera, Plagiostyles africana et Dacryodes buettneri ; la dominance relative des espèces est comprise entre 0,04 et $10,2 \%$ et sa moyenne est égale à $1,15 \pm 0,09 \%$. Plagiostyles africana est l'espèce phare alors que $59 \%$ des taxons ont une valeur inférieure à $1 \% ; 1$ 'importance relative est comprise entre 0,3 et $7,8 \%$ avec une moyenne de $1 \pm 0,07 \%$. Les principales essences sont Santiria trimera, Plagiostyles africana et Dacryodes buettneri ; l'indice de valeur d'importance varie de 0,8 à $23,5 \%$ (soit $3,1 \pm 0,19 \%$ en moyenne). $18 \%$ des taxons présentent une valeur inférieure à $1 \%$; l'indice de valeur d'importance des familles oscille de 0,8 à $52,11 \%$ avec une moyenne de $9,7 \pm 1,42 \%$. Il met en évidence les Euphorbiaceae (52,1\%), Burseraceae $(34,8 \%)$, Fabaceae $(37,5 \%)$, Myristicaceae (16,3\%), Rubiaceae (18,7\%), Sapindaceae $(16,4 \%)$ et Annonaceae $(18,6 \%)$;

- La forêt marécageuse, la fréquence relative varie de 1,1 à $5,3 \%$ alors que sa moyenne est de $1,8 \pm 0,1 \% .82 \%$ des taxons ont une valeur inférieure à $2 \%$; la densité relative varie de 0,5 à $11,3 \%$ et sa moyenne est de $1,8 \pm 0,21 \%$. Les espèces mises en relief sont Allophyllus africanus, Eriocoelum microspermum et Diospyros canaliculata; la dominance relative des espèces oscille de 0,09 à $15,63 \%$ et la moyenne est de $1,96 \pm 0,8 \%$. Mitragyna stipulosa, Allophyllus africanus, Scyphocephalium mannii et Rothmannia lujae sont les espèces les plus importantes alors que $73 \%$ des taxons ont une valeur inférieure à $1 \%$; l'importance relative à une valeur comprise entre 0,5 et $8,6 \%$ (soit une moyenne de $1,8 \pm 0,18 \%)$. Les taxons à forte contribution sont Allophyllus africanus, Eriocoelum microspermum et Diospyros canaliculata, alors que $61 \%$ des taxons ont une valeur en dessous de $1 \%$; l'indice de valeur d'importance varie de 1,6 à $25,7 \%$ (soit $5,44 \pm 0,5 \%$ en moyenne) et met en évidence Allophyllus africanus, Eriocoelum microspermum et Diospyros canaliculata; (vi) l'indice de valeur d'importance des familles variant de 1,6 à $62,9 \%$ avec une moyenne de $12,8 \pm 2,23 \%$. Les familles les plus importantes sont les Rubiaceae $(62,9)$, Sapindaceae (44,3\%), Fabaceae (33,6\%), Burseraceae (17,7\%), Malvaceae (17,5\%), Ebenaceae (17,6\%), Anacardiaceae (17,5\%), Myristicaceae $(18,4)$.

Pour les ligneux de dhp $\geq 70 \mathrm{~cm}$

- La forêt de terre ferme, la fréquence relative varie de 1,1 à $7,5 \%$, alors que la moyenne est de $2,5 \pm 0,22 \%$. Les taxons les plus importants sont Dacryodes buettneri, Petersianthus macrocarpus, Blighia welwitschii, Pentaclethra eetveldeana et Pentaclethra macrophylla; la densité relative est entre 0,6 et $34,1 \%$ et sa moyenne est de 2,5 $\pm 0,33 \%$. Dacryodes buettneri est l'espèce mise en valeur, pendant que $37,5 \%$ de l'inventaire sont en dessous de $1 \%$; la dominance relative des espèces oscille de 0,6 à $10,4 \%$ alors que la moyenne est de $2,5 \pm$ $0,25 \%$. L'espèce dominante est Dacryodes buettneri, alors que $35 \%$ des espèces ont une dominance relative inférieure à $1 \%$; 
l'importance relative à une valeur comprise entre 0,7 et $17,3 \%$. Le taxon à forte contribution est Dacryodes buettneri; l'indice de valeur d'importance varie de 2,2 à $52 \%$ (soit une moyenne $7,5 \pm 0,8 \%$ ) et révèle Petersianthus macrocarpus, Pentaclethra eetveldeana, Pentaclethra macrophylla, Blighia welwitschii, Pterocarpus soyauxii et Scyphocephalium mannii; l'indice de valeur d'importance des familles variant de 2,2 à $56,2 \%$ présente une moyenne de $11,8 \pm$ $1,75 \%$. Les familles prédominantes sont les Fabaceae (49,07\%), Burseraceae $(43,84 \%)$, Myristicaceae (12,08\%), Lecythidaceae $(11,57 \%)$ et Irvingiaceae $(9,87 \%)$. Soulignons que $80 \%$ des espèces recensées ont un indice de valeur d'importance inférieure à $10 \%$, contre $30 \%$ au niveau des familles ;

- La forêt périodiquement inondée, la fréquence relative varie de 1,4 à $8,5 \%$ et la moyenne est de $2,5 \pm 0,21 \%$. Les taxons les plus importants sont Dacryodes buettneri, Scyphocephalium mannii, Klainedoxa gabonensis et Canarium schweinfurthii; la densité relative est entre 1,1 et $11,1 \%$, soit une moyenne de $2,5 \pm 0,25 \%$. Les espèces dominantes sont Dacryodes buettneri, Scyphocephalium mannii, Klainedoxa gabonensis, Mitragyna stipulosa, Petersianthus macrocarpus et Canarium schweinfurthii; la dominance relative des espèces oscille de 0,8 à $10,9 \%(2,5 \pm 0,25 \%$ en moyenne). Dacryodes buettneri est l'espèce mise en relief; l'importance relative à une valeur comprise entre 1,1 et $10,2 \%$, soit $2,5 \pm 0,24 \%$ en moyenne. Les taxons à forte contribution sont Dacryodes buettneri, Scyphocephalium mannii, Klainedoxa gabonensis, Mitragyna stipulosa, et Canarium schweinfurthii; l'indice de valeur d'importance varie de 2,52 à $19,57 \%$ (soit $5 \pm$ $0,5 \%$ en moyenne) et met en évidence Dacryodes buettneri, Scyphocephalium mannii, Klainedoxa gabonensis, et Canarium schweinfurthii; l'indice de valeur d'importance des familles variant de 2,52 à $33,29 \%$ ressort les Burseraceae $(51,5 \%)$,
Myristicaceae (34,6\%), Fabaceae (29,6\%), Irvingiaceae $(24,2 \%)$ et Rubiaceae $(18,1 \%)$; - La forêt marécageuse, la fréquence relative varie de 4,8 à $19 \%$ (soit $7,7 \pm 1 \%$ en moyenne). Les taxons les plus importants sont Mitragyna stipulosa et Scyphocephalium mannii; la densité relative varie de 2,2 à $46,7 \%$ et la moyenne est de $7,7 \pm 1,81 \%$. Mitragyna stipulosa, est l'espèce dominante ; la dominance relative des espèces oscille de 2 à 46,3\% (soit une moyenne de 7,7 $\pm 1,85 \%$ ). L'espèce mise en valeur est Mitragyna stipulosa; (iv) l'importance relative à une valeur comprise entre 3 et $37,3 \%$. Le taxon à forte contribution est Mitragyna stipulosa. Il est suivi de très loin par Scyphocephalium mannii, Coelocaryon botryoïdes et Pseudospondias microcarpa; l'indice de valeur d'importance oscille de 9,9 à $112 \%$ (soit une moyenne de $23,1 \pm 4,6$ ) et met en évidence Mitragyna stipulosa, Scyphocephalium mannii, Coelocaryon botryoïdes et Pseudospondias microcarpa; l'indice de valeur d'importance des familles varie de 9 à $139 \%$ pour une moyenne de $33,3 \%$. Les familles dominantes sont les Rubiaceae (139\%), Myristicaceae (41,62\%), Fabaceae (27,4\%), Anacardiaceae (27,4\%).

\section{Structure diamétrique}

La structure diamétrique des arbres de $10 \leq \mathrm{dhp}<70 \mathrm{~cm}$ révèle une proportion très élevée des tiges de petit diamètre dans les trois types forestiers (Figure 4). Dans la forêt marécageuse, la première classe de diamètre regroupe plus de $2 / 3$ de l'inventaire (Soit $62,43 \%$ ). Ce taux est de $50,63 \%$ dans la forêt de terre ferme et atteint $54,78 \%$ dans le type périodiquement inondé (Figure 4A). Cette tendance se maintient dans l'inventaire des arbres de $\mathrm{dhp} \geq 70 \mathrm{~cm}$, avec notamment la classe sept (Figure 4B). En effet, les essences de première grandeur $(\mathrm{dhp}>100 \mathrm{~cm})$ des forêts du Bassin du Congo étant presque absente du cortège régénératif, seules s'expriment les espèces donnant les essences de seconde grandeur $(70 \leq \mathrm{dhp}<100 \mathrm{~cm})$. Dans le cas présent, les plus représentatifs 
sont Dacryodes buettneri et Santiria trimera. Dans la forêt de terre, $84,89 \%$ des ligneux sont dans la classe $7 ; 54,78 \%$ dans la forêt périodiquement inondée; et $93,33 \%$ dans le type inondé. L'analyse des diamètres moyens par classe de diamètre montre que les ligneux inventoriés sont majoritairement de petit diamètre. En effet, les moyennes sont toutes confinées aux bornes inférieures des classes de diamètres (Tableau 2). La tendance hyperbolique dite encore en « $\mathrm{L}$ » ou en $« \mathrm{~J}$ » renversée des courbes diamétriques présagerait une régénération satisfaisante au sein des trois faciès de la forêt à Marantaceae de Lossi.

\section{Surface terrière}

Les faciès forestiers montrent des surfaces terrières très faibles (Tableaux 1 et 2). Elle est de $9,7 \mathrm{~m}^{2}$ pour 0,5 ha dans le faciès marécageux; de 21,7 et $23,5 \mathrm{~m}^{2}$.ha ${ }^{-1}$ dans les faciès de terre ferme et périodiquement inondé, pour les ligneux de 10 $\leq \mathrm{dhp}<70 \mathrm{~cm}$. Les surfaces terrières des ligneux de $\mathrm{dhp} \geq 70 \mathrm{~cm}$, sont respectivement de $3,8 \mathrm{~m}^{2} \cdot$ ha $^{-1}$ dans le faciès marécageux, de $4,3 \mathrm{~m}^{2} \cdot \mathrm{ha}^{-1}$ dans le périodiquement inondé et de $6,4 \mathrm{~m}^{2} \cdot \mathrm{ha}^{-1}$ dans le faciès de terre ferme. Dans l'ensemble, ces valeurs sont 30 à $40 \%$ en dessous des données des forêts semi décidues d'Afrique centrale dont elle est un maillon.

Quant au rapport de la surface terrière par la densité, il est de 0,05 dans le faciès marécageux, de 0,06 dans le périodiquement inondé et de terre ferme, pour les arbres de 10 $\leq \mathrm{dhp}<70 \mathrm{~cm}$. Ce paramètre est, pour les ligneux de $\mathrm{dhp} \geq 70 \mathrm{~cm}$, de 0,42 dans la forêt marécageuse et de 0,48 dans les faciès périodiquement inondé et de terre ferme. Ces valeurs qui sont nettement inférieure à 1 mettent en exergue une nette dominance des individus de petit diamètre, dans tous les types forestiers.

\section{Spectres bioécologiques}

Les spectres bioécologiques bruts et pondérés se fondent sur l'analyse des diaspores, des types foliaires et phytogéographiques dans les trois faciès forestiers (Figure 5 et 6 ).

\section{Ligneux de $10 \leq d h p<70 \mathrm{~cm}$}

- Dans les trois types forestiers, la distribution des diaspores montrent une dominance des sarcochores. La proportion des sarcochores, dans les spectres brut et pondéré, est supérieure à $80 \%$ (Figures 5A1-A2). Les diaspores anémochores sont faiblement représentées. Dans ces types forestiers, la sarcochorie s'impose comme le mode ultime de dissémination. De facto, elles sont tributaires des animaux, notamment la zoochorie ;

- La distribution des types foliaires révèle une nette dominance numérique des espèces mésophylles ( 92 à $98 \%$ ) dans tous les faciès forestiers (Figure 5B1-B2) ;

- La répartition des types phytogéographiques met en évidence l'élément du centre régional d'endémisme Guinéo-Congolais. Leur proportion varie de 80 à $94 \%$, selon qu'il s'agit du spectre brut ou pondéré, dans les différents faciès (Figure 5C1-C2). Le taux élevé des taxons bas- et centro-guinéen scelle l'appartenance de cette flore à ces deux subcentres d'endémisme de la région Guinéo-Congolaise. Ces faciès sont naturels et subissent moins les influences extérieures.

\section{Ligneux de dhp $\geq 70 \mathrm{~cm}$}

- Les tendances observées avec les ligneux de $10 \leq \mathrm{dhp}<70 \mathrm{~cm}$ se maintiennent dans cette classe de diamètre, souvent en des proportions moindres (Figures 6A1-C2). Il est intéressant de souligner la progression des diaspores anémochores qui dépassent généralement le $1 / 4$ des taxons inventoriés, dans les trois types forestiers. 
Tableau 1 : Synopsis floristique et écologique des ligneux de la forêt à Marantaceae de Lossi.

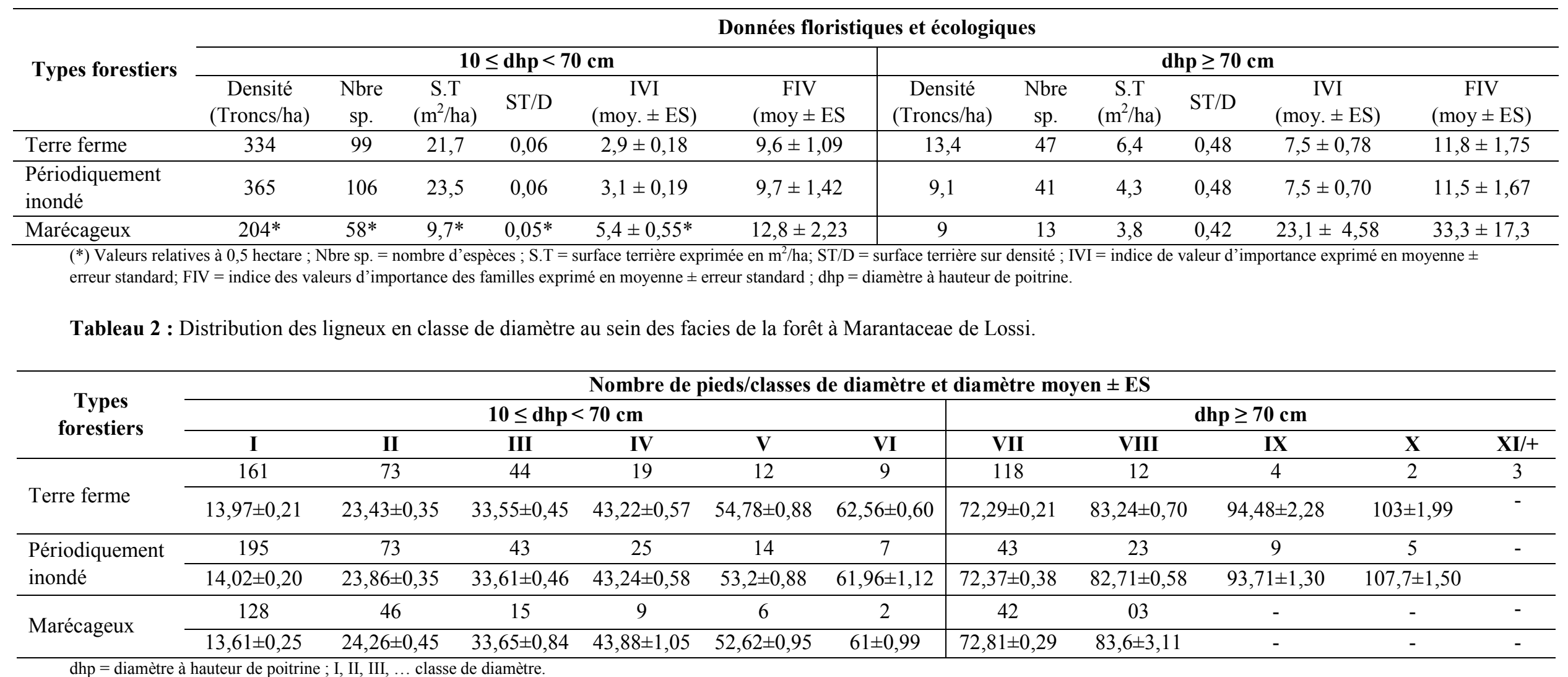




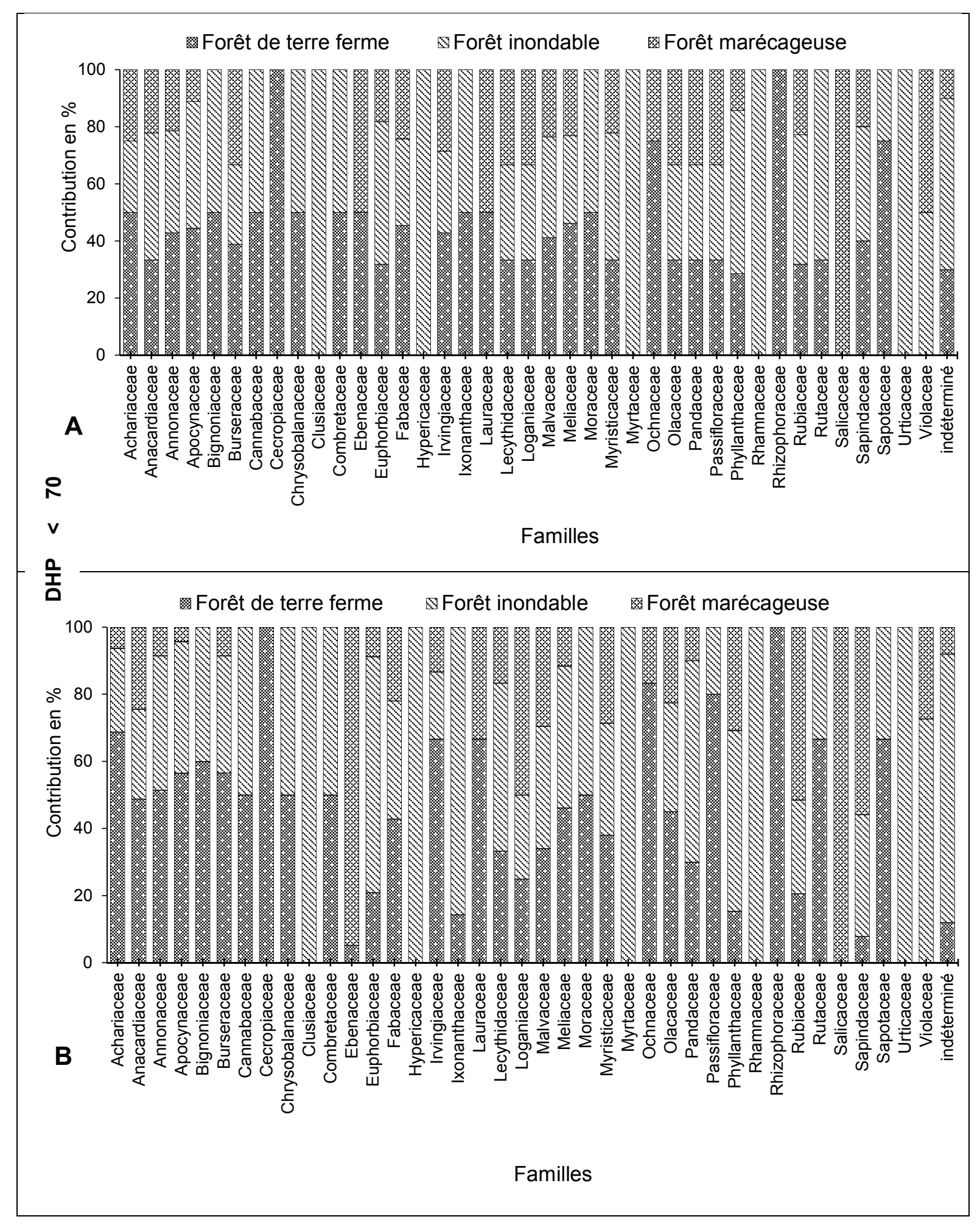




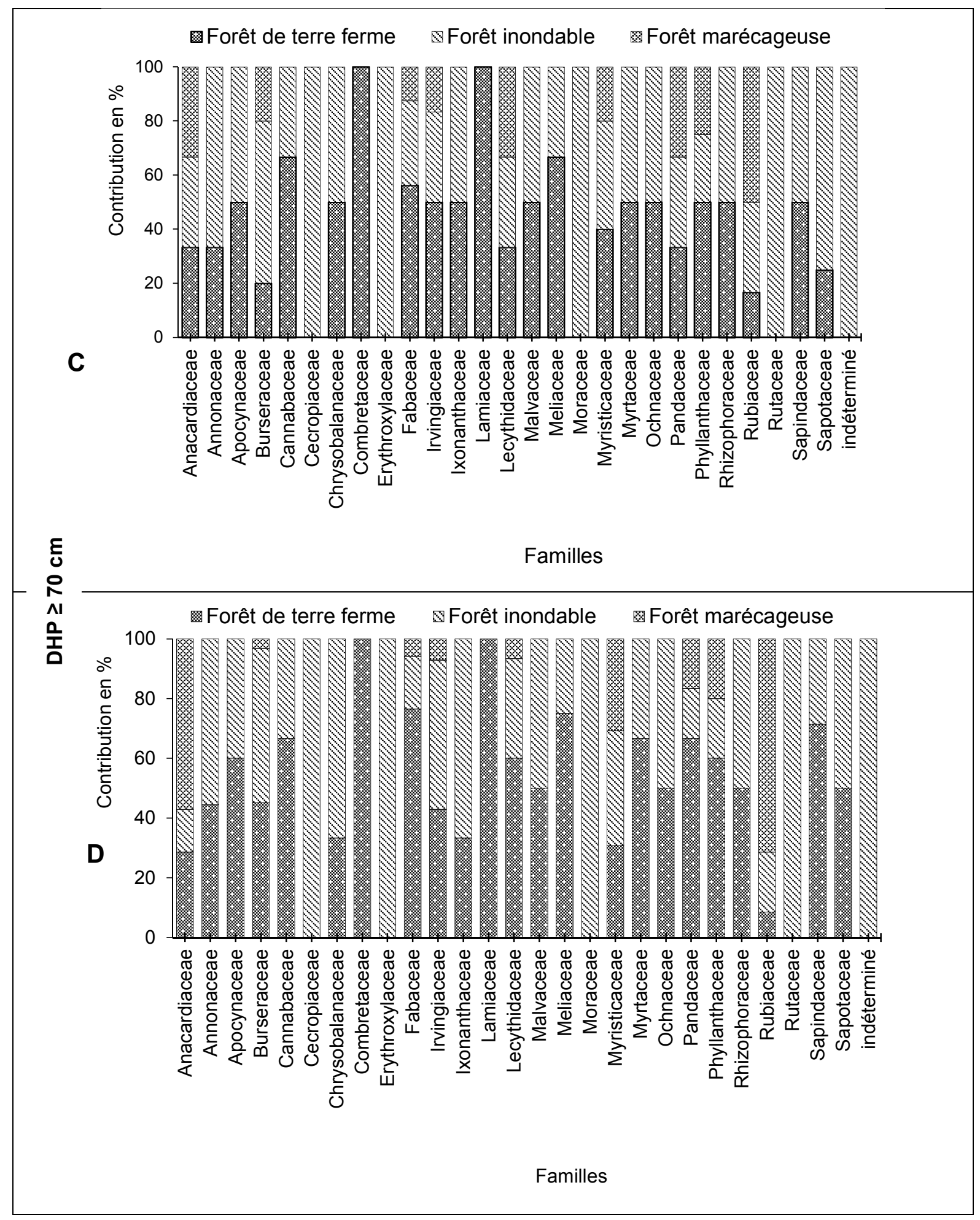

Figure 2 : Spectres brut et pondéré des faciès forestiers à Marantaceae de Lossi. A et $\mathrm{C}=$ spectre brut ; $\mathrm{B}$ et $\mathrm{D}=$ spectre pondéré. 


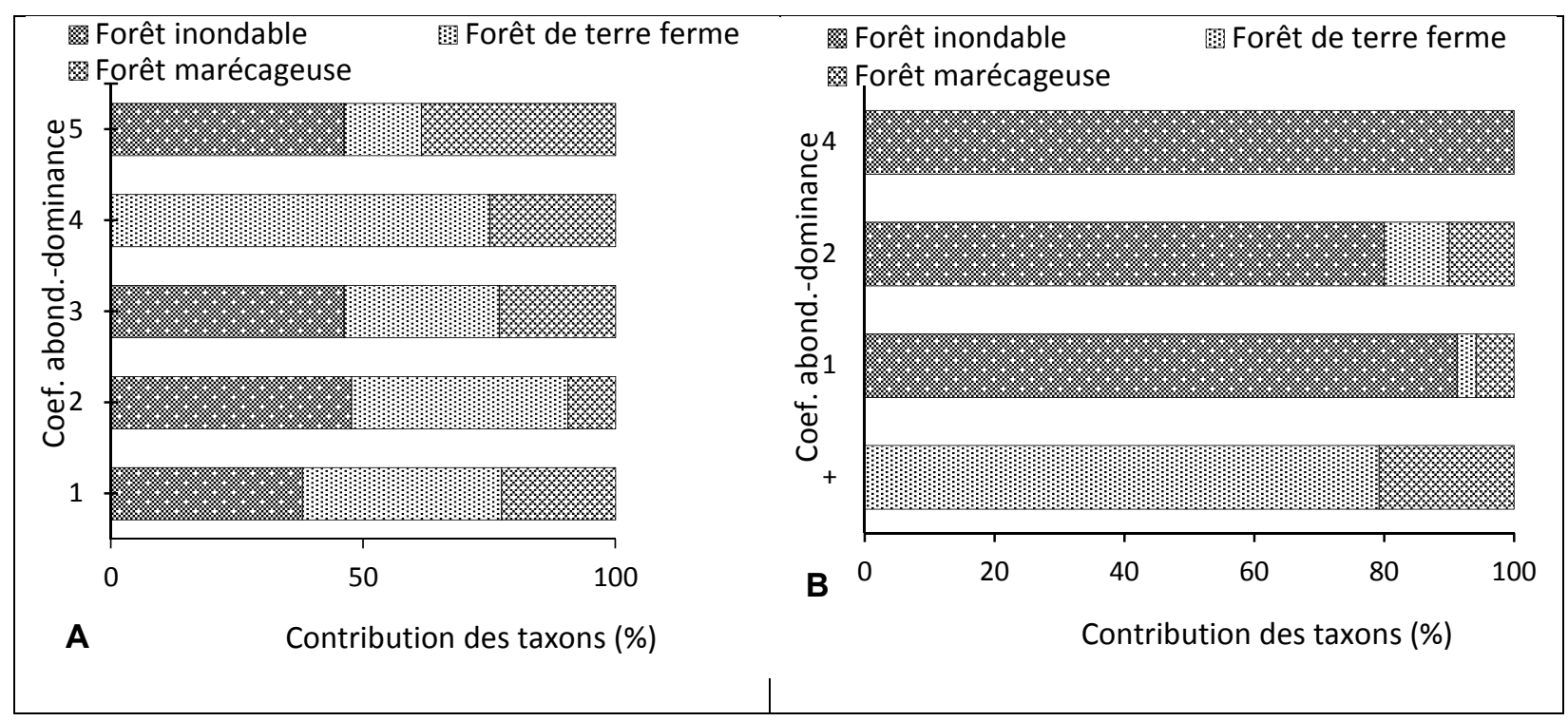

Figure 3 : Coefficient d'abondance-dominance des faciès de la forêt à Marantaceae. $\mathrm{A}=$ arbres de dhp $<70 \mathrm{~cm} ; \mathrm{B}=$ arbres de $\mathrm{dhp} \geq 70 \mathrm{~cm}$.

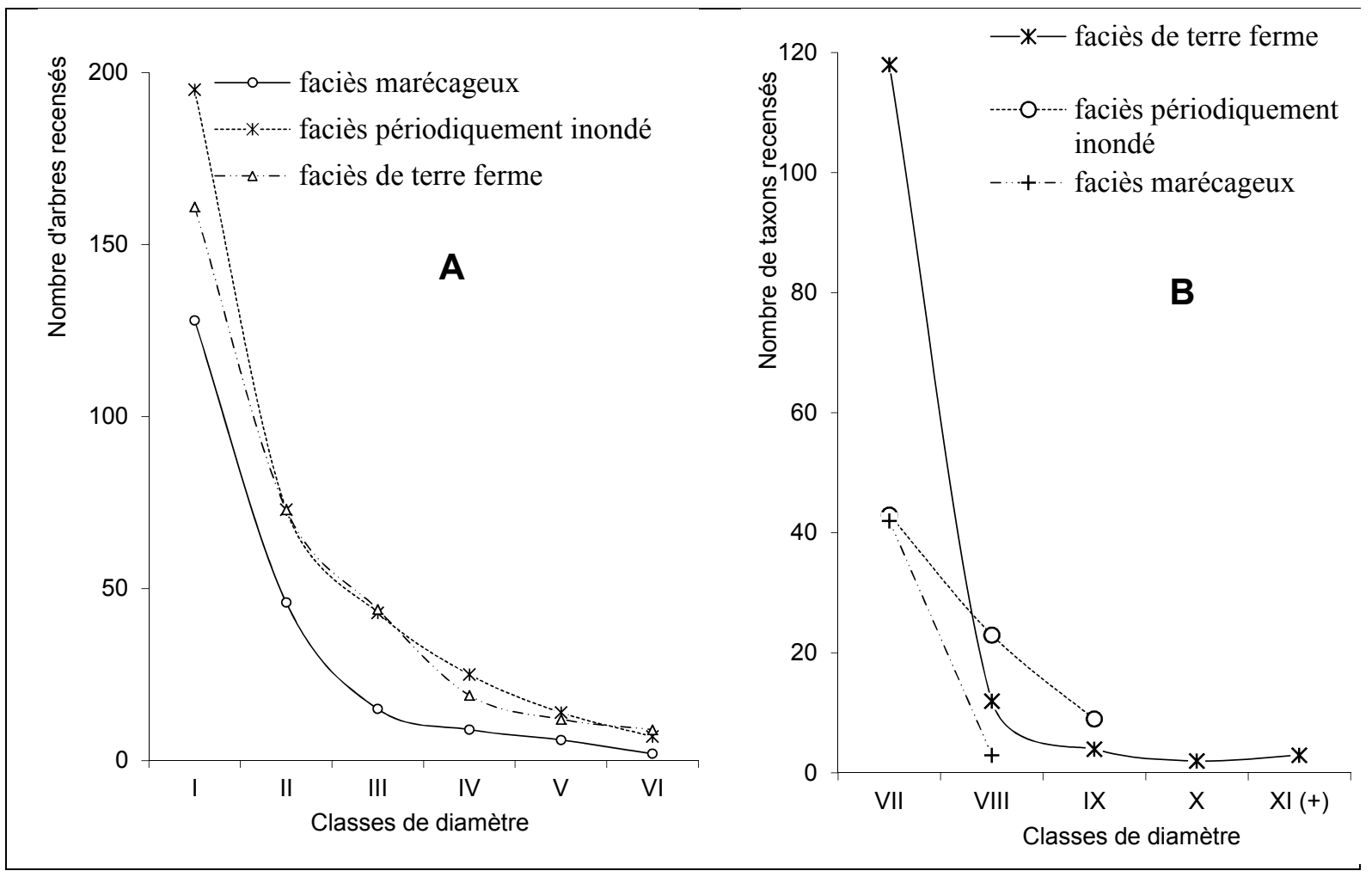

Figure 4: Distribution des ligneux en classes de diamètre dans les types forestiers à Marantaceae. $A=$ Ligneux de $10 \leq \mathrm{dhp}<70 \mathrm{~cm} ; \mathrm{B}=$ Ligneux de $\mathrm{dhp} \geq 70 \mathrm{~cm}$. 


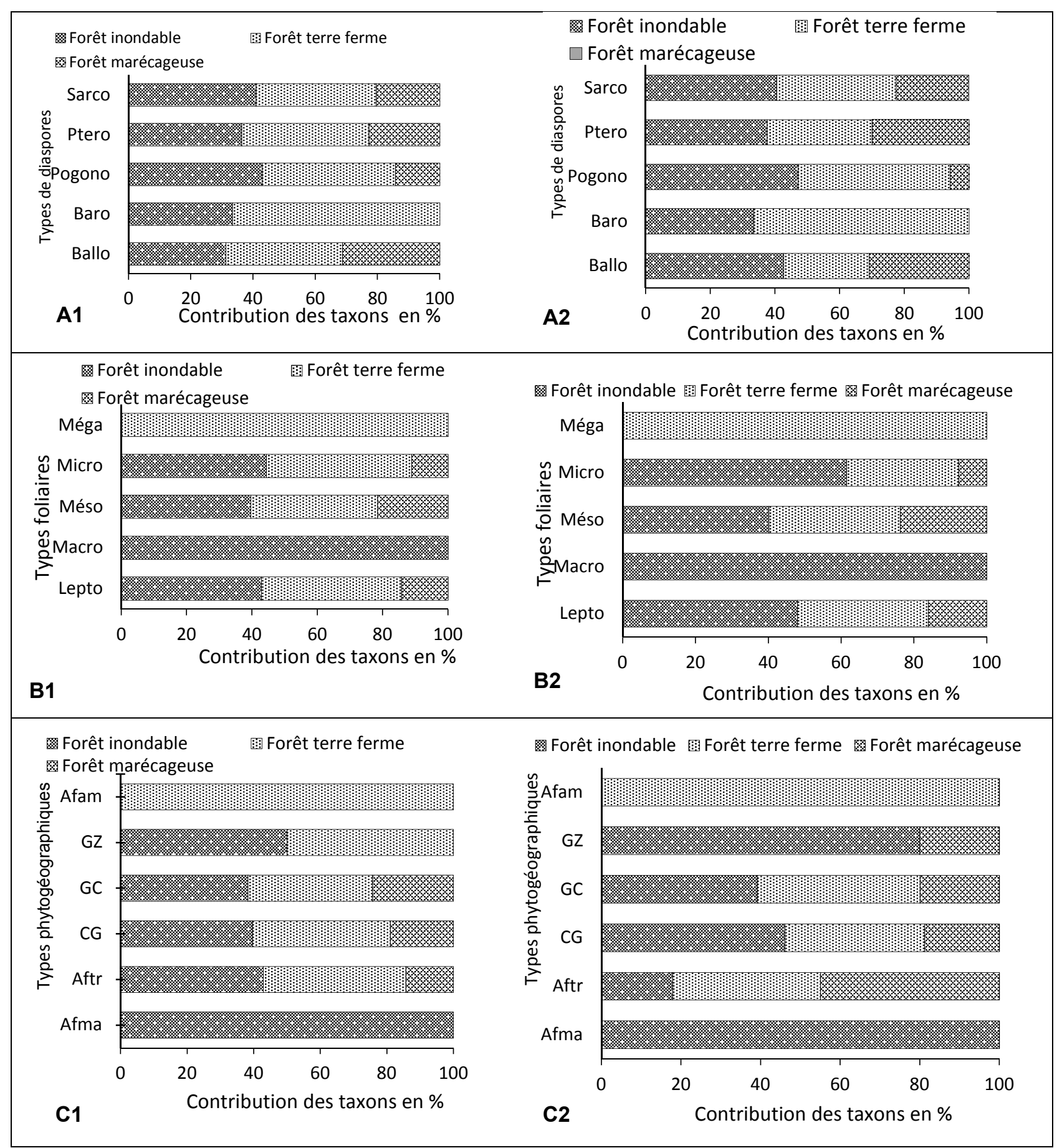

Figure 5: Synoptique des spectres bioécologiques et phytogéographiques des arbres de dhp $<70$ $\mathrm{cm} . \mathrm{A}_{1}, \mathrm{~B}_{1}, \mathrm{C}_{1}=$ spectre brut $; \mathrm{A}_{2}, \mathrm{~B}_{2}, \mathrm{C}_{2}=$ spectre pondéré. 


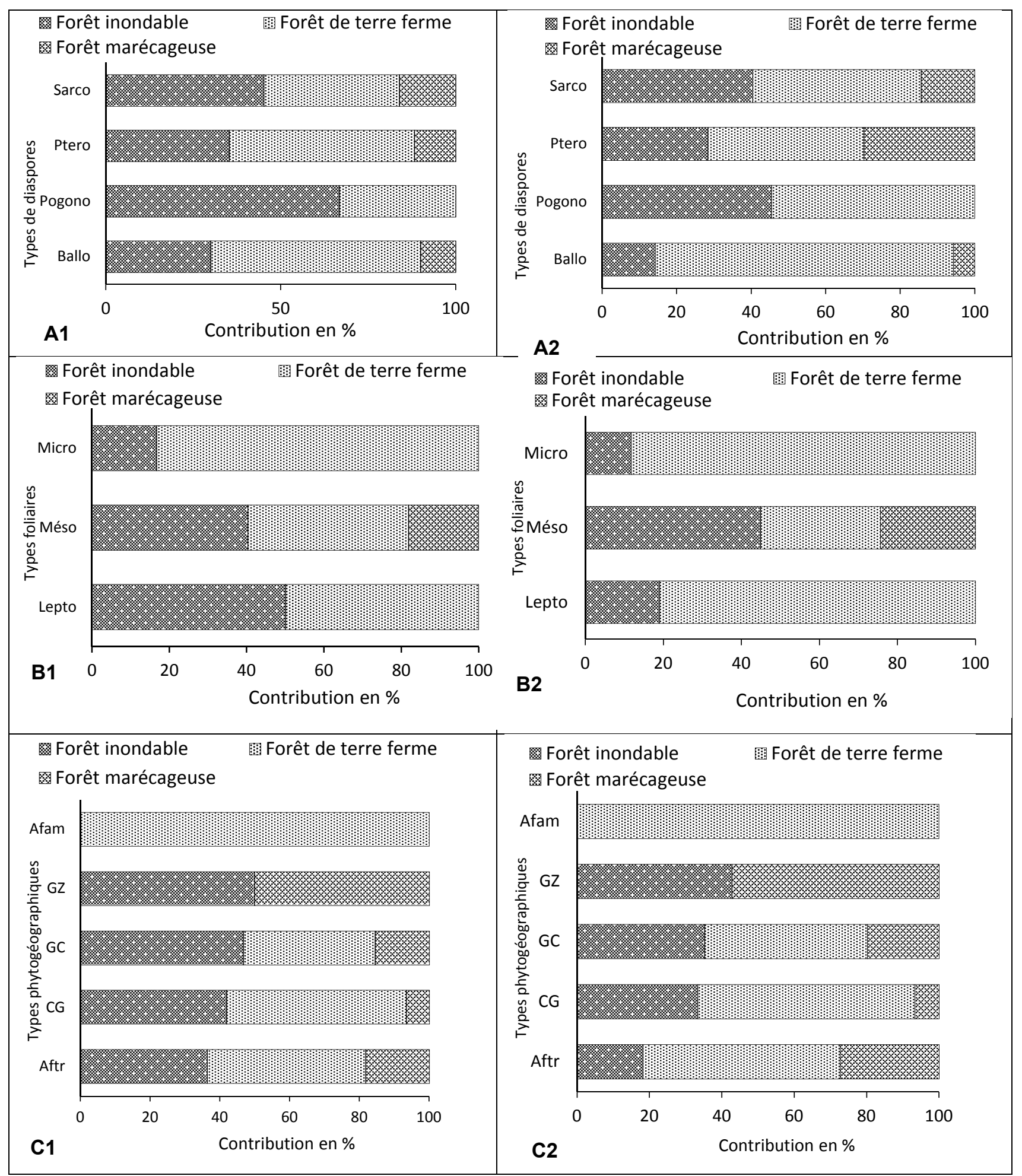

Figure 6: Synoptique des spectres bioécologiques et phytogéographiques des arbres de dhp $\geq 70$ $\mathrm{cm} . \mathrm{A}_{1}, \mathrm{~B}_{1}, \mathrm{C}_{1}=$ spectre brut $; \mathrm{A}_{2}, \mathrm{~B}_{2}, \mathrm{C}_{2}=$ spectre pondéré. 


\section{DISCUSSION}

\section{Analyse de la phytodiversité}

$\mathrm{La}$ richesse floristique des facies forestiers est marquée par la dominance d'au moins une des familles caractéristiques des écosystèmes forestiers semi décidues d'Afrique centrale (Puig, 2001). Parmi ces familles, les Fabaceae, les Rubiaceae et les Euphorbiaceae traduisent leur affinité avec les faciès étudiés par plusieurs auteurs dans le Bassin du Congo: Cusset (1989) dans le massif du Mayombe au Congo ; Kouka (2000, 2006) au Nord Congo; Mosango (1991a, 1991b) en RDC (ex Zaïre) ; Sonké (1998) au Cameroun; Yongo et De Foucault (2001) en RCA. Comparativement au nombre d'espèces d'arbres par hectare des forêts tropicales, pour les arbres de dhp $\geq 10 \mathrm{~cm}$, qui varie de 155 à 313 espèces d'arbres.ha ${ }^{-1}$ suivant les milieux (Puig, 2001), les types forestiers de la forêt à Marantaceae de Lossi sont très faiblement diversifiés. La valeur moyenne d'espèces d'arbres par hectare est de 87,78 $\pm 11,42$ pour les ligneux de $10 \leq \mathrm{dhp}<70 \mathrm{~cm}$ et de $4 \pm 0,46$ pour ceux de dhp $\geq 70 \mathrm{~cm}$. L'explication plausible de ce phénomène est à rechercher dans la structure, la composition et le fonctionnement de la strate herbacée qui ne permet pas la régénération naturelle des ligneux in situ et encore moins les intrusions naturelles des anémochores (Kouka, 2006; Gillet, 2013). En effet, $1 / 4$ des espèces recensées dans la classe des $\mathrm{dhp} \geq 70 \mathrm{~cm}$ sont absentes dans la classe de $10 \leq \mathrm{dhp}<70 \mathrm{~cm}$. Outre ce paramètre, la majorité des espèces caractéristiques de la forêt dense ont des densités extrêmement faibles (1 à 3 arbres.ha $\left.{ }^{1}\right)$. Ces taxons représentent, suivant les types forestiers, 70 à $75 \%$ du total des espèces inventoriées (Lejoly, 1994). Cette donnée est conforme à son qualificatif de forêt clairsemée à Marantaceae (Kouka, 2006 ; Gillet, 2013). La convergence phytogéographique des éléments Atlantique (nigéro-camerounogabonais) et camerouno-congolais au niveau de la Haute Sangha explique l'importance des espèces typiquement Atlantique, notamment Scyphocephalium mannii et le fort taux des
Burseraceae: Dacryodes sp. et Santiria trimera (Kimpouni et al., 2013).

L'analyse de la densité des faciès étudiés révèle un nombre de troncs par hectare relativement faible par rapport aux valeurs connues des forêts tropicales d'Afrique centrale et d'ailleurs. Voisines des densités trouvées par Kimpouni et al. (2008a, 2008b) dans les forêts à Aucoumea klaineana de la région atlantique congolaise, elles sont 30 à $50 \%$ en dessous de celles des forêts tropicales semi décidues. Variant de 450 à 600 arbres.ha $^{-}$ 1 dans les forêts tropicales semi décidues d'Afrique centrale, la densité moyenne des faciès étudiés dans la forêt à Marantaceae de Lossi est de $301 \pm 37,33$ pour la classe des ligneux de $10 \leq \mathrm{dhp}<70 \mathrm{~cm}$ contre $10,5 \pm$ 1,11 pour celle de $\mathrm{dhp} \geq 70 \mathrm{~cm}$.

En couplant la densité des espèces et la composition floristique des ligneux, cette forêt s'identifie comme un faciès à Burseraceae et Euphorbiaceae dont les marqueurs spécifiques sont Santiria trimera (31 arbres.ha $\left.{ }^{-1}\right)$, Dacryodes sp. (22 arbres.ha $\left.{ }^{-1}\right)$ et Plagiostyles africana (23,5 arbres. $\left.\mathrm{ha}^{-1}\right)$. La caractérisation de ce faciès forestier par les Burseraceae (Dacryodes sp. et Santiria trimera) et les Euphorbiaceae (Plagiostyles africana) est soutenue par leur coefficient d'abondance-dominance. Cette physionomie floristique traduit une affinité avec les forêts étudiées par Kouka (2006), dans le Sud du parc national d'Odzala.

La diversité floristique des ligneux de $10 \leq \mathrm{dhp}<70 \mathrm{~cm}$, analysée en fonction des indices des valeurs d'importance, révèle que 50 à $76 \%$ des espèces ont des contributions très faibles qui varient de 0,74 à $1,80 \%$, suivant les types forestiers. Parmi ces espèces, plusieurs d'entre elles sont des essences de première grandeur comme Klainedoxa gabonensis, Canarium schweinfurthii, Chrysophyllum africana, Phyllocosmus africanus, Fillaeopsis discophora, Petersianthus macrocarpus, Lophira alata, Staudtia kamerunensis, etc. Il ressort de cette analyse que le taux des taxons rares est très élevé dans les différents faciès et par conséquent des faibles degrés de similarités 
entre eux. En juxtaposant les données de la fréquence relative, de la densité relative et de la surface terrière, les Burseraceae (Dacryodes buettneri et Santiria trimera) et les Euphorbiaceae (Plagiostyles africana) sont les deux taxons qui caractérisent les faciès périodiquement inondé et de terre ferme. Le type marécageux est dominé par les taxons arbustifs (Allophyllus africanus, Eriocoelum microspermum et Diospyros canaliculata). Les essences d'arbres, notamment de première grandeur, ont une contribution insignifiante et les mieux classées avec un $5 \leq$ IVI $<10$ sont Mitragyna stipulosa, Pseudospondias microcarpa, Berlinia grandiflora, Scyphocephalium mannii et Rothmannia lujae. En effet, seuls ces taxons ont concomitamment une surface terrière, une fréquence et une densité relatives élevées. La prépondérance de l'IVI desdits taxons est associée au nombre élevé d'individus, ce qui traduit un développement grégaire, dans la classe de diamètre de $10 \leq \mathrm{dhp}<70 \mathrm{~cm}$. Les Fabaceae, quoique très diversifiées avec un indice de valeur d'importance de famille (VIF) élevé, n'ont aucun taxon susceptible d'influencer la caractérisation de la flore des types forestiers étudiés dans la forêt à Marantaceae de Lossi. Dans la classe des arbres de dhp $\geq 70 \mathrm{~cm}$, les espèces prépondérantes sont Dacryodes buettneri, Scyphocephalium mannii et Petersianthus macrocarpus, mais leur distribution très éparse n'est pas à même de marquer la caractérisation de la flore.

\section{Caractéristiques structurales de la phytocénose}

Les types foliaires reflétant la capacité photosynthétique des espèces, leur rôle dans la caractérisation de la structure verticale des écosystèmes forestiers n'est plus à démontrer (Puig, 2001; Senterre, 2005; Gillet, 2013). Ainsi, les ligneux se classent en strate supérieure (canopée), en strate moyenne (sous canopée) et en espèces de sous-bois. La nette prépondérance des mésophylles sur les autres types foliaires, s'explique par la structure de cette formation ligneuse à Marantaceae dont les cimes ne sont pas jointives au niveau de toutes les strates. Ce phénomène qui induit une sélection naturelle au niveau de la flore (Senterre, 2005; Gillet, 2013), favorise le développement des mésophylles (plantes héliophytes) par rapport aux autres types plus exigeant écologiquement (espèces sciaphytes).

Les données de la surface terrière révèlent que les faciès étudiés, dans la forêt à Marantaceae de Lossi, ont une projection des sections des troncs au sol très faible. Par rapport aux données des forêts denses semi décidues d'Afrique centrale et d'ailleurs, cette surface terrière est de l'ordre de 22,6 $\pm 0,6$ $\mathrm{m}^{2} \cdot \mathrm{ha}^{-1}$ en moyenne, pour les individus de 10 $\leq \mathrm{dhp}<70 \mathrm{~cm}$, dans les faciès de terre ferme et périodiquement inondé. Cette valeur correspond à la limite inférieure des forêts denses semi décidues d'Afrique centrale. En effet, ces valeurs sont 2 à 3 fois en dessous des données collectées dans la région (Puig, 2001 ; Dibong et al., 2003 ; Sunderland et al., 2003 ; Nusbaumer et al., 2005 ; Kenfack et al., 2007 ; Frontier et al., 2008 ; Kimpouni et al., 2008 ; Priso et al., 2010 ; Vroh et al., 2010 ; Chuyong et al., 2011 ; Gonmadje et al., 2011). Elle est de $5,34 \mathrm{~m}^{2} \cdot \mathrm{ha}^{-1}$ pour ceux de dhp $\geq 70$ $\mathrm{cm}$. En rapport avec les faibles densités relevées dans cette forêt, la surface terrière montre que les arbres ont un dhp assez modeste. L'étude de la dispersion des taxons en classes de diamètre conduit à une courbe de distribution hyperbolique. Elle traduit un niveau de régénération naturelle suffisant des ligneux, conséquence d'un recrutement régulier au sein des classes de diamètre (Puig, 2001). Toutefois, l'analyse critique de la cohorte régénérative démontre que la régénération naturelle de ces faciès est nettement insuffisante (Van Loon, 2009; Gillet, 2013). En effet, le cortège floristique des classes de diamètre 1 et 2 ont un taux élevé de ligneux dont les espèces ne contribuent pas à l'émergence des essences de première grandeur. Suivant le type forestier, les ligneux de $10 \leq \mathrm{dhp}<70 \mathrm{~cm}$, ce taux varie de 51,55 à $77,34 \%$ des arbres recensés dans la classe de diamètre 1. Dans la classe de diamètre 2 , ce taux oscille entre 35,62 et 
$65,22 \%$ des ligneux ; alors qu'il est compris entre 46,67 et $54,54 \%$ des ligneux recensés dans la classe de diamètre 3 . Le passage d'une classe de diamètre à une autre ayant pour corollaire la baisse de la densité des ligneux (Puig, 2001), on note une faible accumulation des individus de gros diamètre au niveau des classes supérieures et la disparition de leur cortège régénératif.

L'indice écologique découlant du rapport S.T/D renseigne sur la dynamique de l'évolution diamétrique et la structure de la population, et le changement de la composition floristique en mettant en valeur les individus les moins représentés, tout en caractérisant non seulement les taxons de gros diamètre. C'est ainsi qu'il est un indicateur de la succession et de l'évolution des ligneux (Kimpouni et al., 2008b). Les valeurs de cet indice étant inférieures à 1 , quel que soit le faciès étudié dans la forêt à Marantaceae de Lossi, il souligne une ascendance des individus de faible diamètre issus des apports spontanés. L'installation $\mathrm{du}$ cortège régénératif est corrélée au changement de la composition floristique des ligneux.

\section{Affinités phytogéographiques de la flore}

L'importance de l'élément caractéristique du centre d'endémisme Guinéo-Congolais et singulièrement la présence des taxons du subcentre d'endémisme Bas-Congolais, authentifie l'appartenance des faciès étudiés aux forêts denses tropicales du Bassin du Congo (White, 1979, 1986 ; Kimpouni et al., 2012). En effet, cet élément endémique est prépondérant tant au niveau des tiges que des espèces. Son importance varie entre 82 et $92 \%$ pour les arbres de $10 \leq \mathrm{dhp}<70 \mathrm{~cm}$ et de 80 à $94 \%$ pour ceux de dhp $\geq 70 \mathrm{~cm}$. L'analyse phytogéographique établit une affinité avec les forêts Atlantiques hyperhumides de l'aire nigéro-camerouno-gabonaise. Cette affinité est associée aux marqueurs comme Scyphocephalium mannii (Myristicaceae) et, Dacryodes sp. et Santiria trimera (Burseraceae). Cette observation dénote que l'élargissement de l'élément de base
Atlantique de l'aire nigéro-camerounogabonaise souligné au niveau du Nord Gabon par Saint-Aubin (1963) étend son influence sur la flore la Haute Sangha (Kimpouni et al., 2013). Cet inventaire donne la preuve de la continuité de cet élément jusqu'au Sud de la Haute Sangha. La flore de ce district phytogéographique évoluerait sous l'influence de l'élément Atlantique de l'aire nigérocamerouno-gabonaise au Sud et de l'élément Bas-Guinéen de l'aire camerouno-congolaise au nord (Kouka, 2006 ; Kimpouni et al., 2013, 2014).

\section{Dissémination des diaspores}

L'analyse des types des diaspores marque une nette dominance des sarcochores dans tous les trois faciès de la forêt à Marantaceae. Ainsi, la manifestation de la zoochorie (notamment l'endozoochorie) se présente comme le mode de dissémination majeur des diaspores à Lossi. Ce résultat est conforme aux études menées dans divers écosystèmes forestiers à travers le monde tropical, comme le montre les travaux de Kimpouni et al. (2012), Gillet (2013) dans les forêts du Bassin du Congo; de Howe \& Vande Kerckhove (1981), de Howe (1983, 1984, 1986) et de Howe et al. (1985) et de Whitney et al. (1998) en région néotropicale. Au plan floristique, ce résultat est le signe d'un haut degré de diversification et d'hétérogénéité atteint par l'écosystème (Puig, 2001). Il est le résultat d'un processus de co-évolution et de mutualisme entre les plantes et les agents de dissémination (Puig, 2001). Ce stade évolutif se caractérise par une forte régression des densités et du nombre d'espèces à diaspores dispersées par les courants aériens (anémochores). L'anémochorie étant un mode de dissémination associée aux espèces héliophiles à tendance souvent pionnières, elle est de ce fait le symbole des phytocénoses jeunes (Puig, 2001). Les données de la dissémination des diaspores couplées à l'inventaire floristique définissent une dynamique floristique très évoluée. La forte diversité floristique enregistrée attribue à cette 
formation forestière une stabilité au stade actuel de son développement. C'est ainsi qu'une modification du milieu aura un faible impact sur son fonctionnement. Il est le symbole d'une relation positive entre la biodiversité et la résilience (Giller et O'Donovan, 2002; Bond et Chase 2002; Wallington et al., 2005 ; Caldeira et al., 2005 ; Mikkelson 2009).

\section{Distribution spatiale de la flore}

Les niches écologiques étant sous la dépendance des conditions du milieu (notamment édaphique), la distribution spatiale des taxons est assujettie aux mécanismes d'adaptations (Puig, 2001). Les données écologiques et les adaptations relevées sur les arbres inventoriés montrent que cette forêt présente plus d'un faciès de végétation. Dans l'ensemble, les trois niches écologiques liées à la balance hydrique du substratum sont remarquables par la composition floristique.

- La forêt de terre ferme est le domaine de Canarium schweinfurthii, Oncoba welwitschii, Musanga cecropioides, Xylopia hypolampra, Pentaclethra sp., Piptadeniastrum africanum, Dacryodes buettneri, Scyphocephalium mannii, Plagiostyles africana, etc.

- La forêt inondable est symbolisée par Symphonia globulifera, Coelocaryon botryoides, Lophira alata, Uapaca sp., Chlamydocola chlamydantha, etc.

- La forêt marécageuse est caractérisée par Mitragyna stipulosa, Rothmannia lujae, Cleistopholis patens, Rinorea oblongifolia, Sarcocephalus pobeguinii, Pseudospondias microcarpa, Berlinia grandifolia, etc.

Suivant les niches écologiques et les adaptations des espèces, on relève deux types de distribution qui sont les modèles grégaire et hasard (Puig, 2001). La diversité floristique étant le reflet de la stabilité fonctionnelle du système, son importance relevée au sein des trois niches la garantie. Cependant, l'analyse du mode de répartition horizontale de la flore associée à l'abondance-dominance atteste un degré de résilience très divergent dans les trois habitats. En effet, leur capacité à amoindrir l'effet induit d'une perturbation du milieu est variable (Loreau et al., 2002 ; Bond et Chase, 2002; Giller et O’Donovan, 2002; Wallington et al., 2005 ; Caldeira et al., 2005 ; Mikkelson, 2009). Elle est plus élevée dans la niche de terre ferme que dans la forêt marécageuse et inondable.

\section{Conclusion}

La forêt à Marantaceae de Lossi est sous l'influence de l'élément atlantique de l'aire nigéro-camerouno-gabonaise. Elle présente une composition floristique typique caractérisée par deux paramètres évoluant en sens opposé, quel que soit le faciès. Il est relevé un haut niveau de diversification auquel s'oppose une faible richesse floristique (pauvreté en essences ligneuses). Quoiqu'étant un maillon des forêts dense d'Afrique centrale, les paramètres de structure des faciès étudiés dans cette forêt confirment leurs spécificités vis-à-vis des forêts denses semi décidues. Leurs valeurs sont généralement 3 à 5 fois inférieures par rapport à l'intervalle des gammes des données connues des écosystèmes forestiers semi décidues de la région. Ces éléments confirment le qualificatif de « forêt clairsemée à Marantaceae » ou encore de « forêt claire à arbres clair-plantés ». L'analyse de la structure diamétrique des ligneux et $\mathrm{du}$ cortège régénératif, dans les trois faciès, montre que le maintien de ces formations, sans les animaux et notamment les grands primates et l'éléphant, ne serait pas garantie. Il est noté que plusieurs espèces des essences de première grandeur ont des densités de 1 à 3 arbres.ha ${ }^{-1}$. Nonobstant cette faible densité, ces taxons qui sont distribués dans les classes de diamètre élevé (assez modeste) n'ont presque pas de sujets dans les premières classes.

\section{CONFLITS D'INTERETS}

Les auteurs déclarent qu'il n'y a aucun conflit d'intérêt. 


\section{RÉFÉRENCES}

APG III. 2009. An update of the Angiosperm phylogeny group classification for the orders and families of flowering plants: APG III. Bot. J. Linn. Society, 161: 105121. DOI: http://dx.doi.org/10.1111/ j.1095-8339.2009.00996.x

Bégué L.1967. Chronique phytogéograhique : les forêts du Nord de la République du Congo (Brazzaville). Bois For. Trop., 111: 63-76.

Bond EM, Chase JM. 2002. Biodiversity and ecosystem functioning at local and regional spatial scales. Ecology Letters, 5: $467-470$.

Caldeira MC, Hector A, Loreau M, Pereira JS. 2005. Species richness, temporal variability and resistance of biomass production in a Mediterranean grassland. Oikos, 110: 115-123.

Cusset G. 1989. La flore et la végétation du Mayombe congolais, état des connaissances. In Revue de Connaissances sur le Mayombe, Sénéchal J, Kabala M, Fournier F (eds). Unesco: Brazzaville; 103-136.

Dallmeier F. 1992. Long-Term Monitoring of Biological Diversity in Tropical Forest Areas, Method of Establishment and Inventory of Permanent Plots. MAB, Digest 11, UNESCO: Rome.

Dansereau P, Lems K. 1957. The Grading of Dispersal Types in Plant Communities and their Ecological Significance. Institut de Botanique, Université de Montreal : Montreal 71; 1-52.

De Wasseige C, De Marcken P, Bayol N, Hiol Hiol F, Mayaux Ph, Desclée B, Nasi R, Billand A, Defourny P, Eba'a Atyi R. 2012. The Forests of the Congo Basin State of the Forest 2010. Publications Office of the European Union: Luxembourg.

De Wasseige C, Flynn J, Louppe D, Hiol Hiol F, Mayaux Ph. 2014. Les Forêts $d u$ Bassin du Congo: Etat des Forêts 2013. Weyrich : Bruxelles.

Évrard C. 1968. Recherches Ecologiques sur le Peuplement Forestier des Sols
Hydromorphes de la Cuvette Centrale Congolaise. Publ. INEAC n ${ }^{\circ} 110$ : Bruxelles.

Frontier S, Pichod-Viale D, Leprêtre A, Avoult D, Luczak C. 2008. Ecosystèmes : Structure, Fonctionnement, Evolution (4 $4^{\mathrm{e}}$ édn). Dunod: Paris.

Giller PS, O’Donovan G. 2002. Biodiversity and ecosystem function: do species matter? Biology and Environment, Proceeding of the Royal Irish Academy, 1028(3): 129-139.

Gillet J-F. 2013. Les forêts à Marantaceae au sein de la mosaïque forestière du Nord de la République du Congo: origines et modalités de gestion. Thèse de Doctorat, Université de Liège - Gembloux Agrobio Tech, Liège, p. 194.

Gonmadje CF, Doumenge C, McKey D, Tchouto GPM, Sunderland $\mathrm{TCH}$, Balinga MPB, Sonke B. 2011. Tree diversity and conservation value of Ngovayang's lowland forests, Cameroon. Biodiversity and Conservation, 20(12): 2627-2648. DOI: http://dx.doi.org/10.1007/s10531-0110095-z

Hecketsweiler P, Doumenge C, Mokoko Ikonga J. 1991. Le Parc National d'Odzala, Congo. UICN : Gland.

Hecketsweiler P, Mokoko-Ikonga J. 1991. La Réserve de Conkouati: Congo, le Secteur Sud-Est. UICN: Gland.

Hecketsweiler P. 1990. La Conservation des Ecosystèmes Forestiers du Congo. UICN: Gland.

Howe HF, Schupp EW, Westley LC. 1985. Early consequences of seed dispersal for a Neotropical tree (Virola surinamensis). Ecology, 66: 781-91.

Howe HF, Vande Kerckhove GA. 1981. Removal of wild nutmeg (Virola surinamensis) crops by birds. Ecology, 62: 1093-1106.

Howe HF. 1983. Annual variation in a Neotropical seed-dispersal system. In Tropical Rain Forest: Ecology and Management, Sutton SL, Whitmore TC, 
Chadwick AC (eds). Oxford University Press: Oxford; 211-227.

Howe HF. 1984. Implications of seed dispersal by animals for tropical reserve management. Biological Conservation, 30: $261-281$.

Howe HF. 1986. Consequences of seed dispersal by birds: a case study from Central America. Journal of the Bombay Natural History Society, 83: 19-42.

Howe HF. 1990. Seed dispersal by birds and mammals: implications for seedling demography. In Reproductive Ecology of Tropical Forest Plants, Bawa KS, Hadley M (eds). Paris: Paris; 191-218.

Kimpouni V, Apani E, Motom M. 2012. Caractéristiques écologiques et composition de la flore ligneuse de la région de Mindouli (Congo). Journal de Botanique, Soc. Bot. France, 57 : 37-47.

Kimpouni V, Loumeto J, Mizingou J. 2008a. Diversité floristique du faciès forestier à Aucoumea klaineana (okoumé) du littoral congolais. Acta Bot. Gallica, 155(3): 323-334. DOI: http://dx.doi.org/10.1080/12538078.2008 .10516113

Kimpouni V, Loumeto J, Mizingou J. 2008b. Diversité floristique du peuplement ligneux de la forêt monodominante à okoumé (Aucoumea klaineana P.) du littoral congolais. Ann. Univ. Marien Ngouabi, 9: 8-20.

Kimpouni V, Motom M, Apani E. 2013. Analyse phytoécologique de la flore ligneuse de la Haute Sangha (Congo Brazzaville). Adansonia, 35(1): 107-134. DOI: http://dx.doi.org/10.5252/a2013n1a9

Köppen W. 1936. Das geographische System der Klimate. In Handbuch der Klimatologie, Köppen W, Geiger R (eds): 1-44.

Kouka LA. 2000. Recherches sur la flore, la structure et la dynamique des forêts du parc national d'Odzala (Congo Brazzaville). Thèse de Doctorat, Université Libre de Bruxelles, Bruxelles, p. 372 .
Kouka LA. 2001. Biotopes et diversité des groupements phytogéographiques dans la flore du parc national d'Odzala (CongoBrazzaville). Syst. Geogr. Pl., 71: 827835. DOI: http://dx.doi.org/10.2307/ 3668721

Kouka LA. 2006. Étude floristique des forêts $\mathrm{du}$ parc national d'Odzala (Congo Brazzaville). Acta Bot. Gallica, 53: 4981. DOI : http://dx.doi.org/10.1080/ 12538078.2006.10515522

Lacoste A, Salanon R. 2006. Éléments de Biogéographie et d'Ecologie: une Compréhension de la Biosphère par l'Analyse des Composantes Majeures des Ecosystèmes. Armand Colin : Paris.

Lejoly J. 1994. Mise en place des transects en vue des inventaires de biodiversité dans le parc national d'Odzala (Congo). Projet ECOFAC - cellule de coordination. Bruxelles, MPEP AGRECO-CTFT.

Loreau M, Downing A, Emmerson M, Gonzalez A, Hughes J, Inchausti P, Joshi J, Norberg J, Sala O. 2002. A new look at the relationship between diversity and stability. In Biodiversity and Ecosystem Functioning: Synthesis and Perspectives, Loreau M, Naeem S, Inchausti J (eds). Oxford University: Oxford; 79-91.

Martin GJ. 2004. Ethnobotany: a Methods Manuel. Earthscan: London.

Mikkelson GM. 2009. Diversity-stability hypothesis. Accessible au http://webpages.mcgill.ca/staff/Group3/g mikke/web/dsh.pdf

Mosango M. 1991a. La forêt secondaire âgée à Zanthoxylum gilletii de l'île Kongolo (Zaïre). Belg. Journ. Bot., 124(2): 152166.

Mosango M. 1991b. Contribution à l'étude botanique et géochimique de l'écosystème forêt en région équatoriale (île Kongolo - Zaïre). Belg. Journ. Bot., 124(2): 167-194.

Nusbaumer L, Gautier L, Chatelain C. 2005. Structure et composition de la Forêt Classée du Scio (Côte d'Ivoire) Etude 
descriptive et comparative. Candollea, 60 (2): 393-443.

Priso RJ, Din N, Dibong SD, Taffouo VD, Kamdem JP, Tchachou M, Amougou A. 2010. Biodiversité et paramètres de structure dans la réserve forestière $d u$ bois des singes et la zone forestière de Bangué (Douala, Cameroun). In Systématique et Conservation des Plantes Africaines, van der Burgt X, Van der Maesen J, Onana J-M (eds). Royal Botanic Gardens: Kew; 265-272.

Puig H. 2001. La Forêt Tropicale Humide. Belin: Paris.

Raunkiaer C. 1934. The Life Forms of Plants and Statistical Plant Geography. Clarendon Press Oxford : London.

Rollet B. 1964. Introduction à l'inventaire forestier du Nord - Congo : Rapport au Gouvernement de la République du Congo ${ }^{\circ}$ 1782. FAO : Rome.

Saint-Aubin (de) GP. 1963. La Forêt $d u$ Gabon. CTFT: Paris.

Samba G, Nganga D, Mpounza M. 2008. Rainfall and temperature variations over Congo-Brazzaville between 1950 and 1998. Theoretical and Applied Climatology, 91: 85-97. http://dx.doi.org/10.1007/s00704-0070298-0

Samba G, Nganga D. 2011. Rainfall variability in Congo-Brazzaville: 19322007. International Journal of Climatology, 32: $\quad$ 854-873. http://dx.doi.org/10.1002/joc.2311.

Senterre B. $2005 . \quad$ Recherches méthodologiques sur la typologie de la végétation et la phytogéographie des forêts denses d'Afrique tropicale. Thèse de Doctorat, Université Libre de Bruxelles, Bruxelles, p. 456.

Sonke B. 1998. Étude floristique et structurale des forêts de la réserve de faune du Dja (Cameroun). Thèse de doctorat, Université Libre de Bruxelles, Bruxelles, p. 256.
Tutin CEG, Williamson EA, Rogers ME, Fernandez M. 1991. A case study of a Plant-animal relationship: Cola lizae and lowland gorillas in the Lope Reserve, Gabon. Journal of Tropical Ecology, 7: 181-199.

Van Loon T. 2009. Projet - Régénération ligneuse dans les forêts claires à Marantacées. Rapport interne. Industrie Forestière de Ouesso, République du Congo : Brazzaville.

Vennetier P. 1977. République Populaire du Congo. Les atlas Jeune Afrique. Éditions J.A. : Paris.

Vroh Bi Tra A, Adou Yoa CY, Kouame D, N'da Dibi H, N'guessan E K. 2010. Diversité floristique et structurale sur le site d'une réserve naturelle volontaire à Azaguié, Sud-est de la Côte d'Ivoire. European Journal of Scientific Research, 45(3): 411-421.

Wallington TJ, Hobbs RJ, Moore SA. 2005. Implications of current ecological thinking for biodiversity conservation: a review of the salient issues. Ecology and Society, 10(1): 15 (online) URL. http://www.ecologyandsociety.org/vol10 /iss1/art15/

White F. 1979. The Guineo-congolian region and its relationships to other phytochoria. Bull. Jard. Bot. Nat. Belg., 49(1/2): 11-55.

White F. 1986. La végétation de l'Afrique. Mémoire Accompagnant la Carte de Végétation de l'Afrique. UNESCO/AETFAT/UNSO, ORSTOM: Paris.

Whitney KD, Fogiel MK, Lamperti AM, Holbrook KM, Stauffer DJ, Hardesty BD, Parker VT, Smith TB. 1998. Seed dispersal by Ceratogymna hornbills in the Dja Reserve, Cameroon. Journal of Tropical Ecology, 14: 351-371.

Yongo O, de Foucault B. 2001. Analyse phytogéographique de la forêt de Ngotto (République Centrafricaine). Syst. Geogr. Pl., 71: 847-857. 